\title{
ANOMALÍAS DE CIRCULACIÓN ATMOSFÉRICA EN 500 Y 1000 HPA ASOCIADA A LA SEQUÍA PRODUCIDA EN LA ARGENTINA DURANTE ENERO DE 2003 A MARZO DE 2004.
}

\author{
ADELIA PERLA ALESSANDRO \\ Dto. de Ciencias de la Atmósfera y los Océanos. FCEN -Universidad de Bs. As. \\ perla@at1.fcen.uba.ar
}

Recibido Diciembre 2003 - Aceptado Noviembre 2007

\begin{abstract}
RESUMO
Neste trabalho se analisa e descreve a situação sinótica associada à precipitação e temperatura do território argentino durante o ano de 2003 e o primeiro trimestre de 2004. A escolha deste período obedece à falta de precipitações em pelo menos dez províncias argentinas, o que ocasionou grandes prejuízos econômicos no setor agropecuário. Com o intuito de determinar as causas deste déficit de precipitações foram analisados os campos médios de altura geopotencial nos níveis de 500 e 1000 $\mathrm{hPa}$ e suas anomalias.

Em 2003, o déficit de precipitação se associa principalmente às anomalias de geopotencial no nível de $500 \mathrm{hPa}$, que mostram um fluxo dominante de SO que atravessa o país diagonalmente entre $45^{\circ} \mathrm{S}$ e $30^{\circ} \mathrm{S}$. Em $1000 \mathrm{hPa}$ o anticiclone subtropical do Atlântico se encontra enfraquecido, deslocado para o norte e afastado do continente, enquanto que o anticiclone subrtopical do Pacífico está mais intenso e deslocado para leste respeito da sua posição normal. No primeiro trimestre de 2004 a seca se deve principalmente à presença de uma crista no nível de $500 \mathrm{hPa}$ posicionada no centro do país.

As anomalias médias de temperatura do período considerado resultam positivas sobre toda a Argentina. Em 2003, essas anomalias se devem à contribuição do fluxo do N-NE desde o norte do país, à subsidência ocasionada pelo anticiclone do Pacífico mais deslocado para o interior do continente e a orografia. No primeiro trimestre de 2004 as anomalias se originam devido ao fluxo do norte e à freqüente circulação anticiclônica no Atlântico ao sul de $35^{\circ} \mathrm{S}$.
\end{abstract}

Palavras- chave: déficit, precipitação, temperatura

\section{ABSTRACT: ANOMALIES OF THE ATMOSPHERIC CIRCULATION AT 500 AND 1000 HPA ASSOCIATED WITH THE DRY PERIOD IN ARGENTINA FROM JANUARY 2003 TO MARCH 2004}

This paper analyzes and describes the synoptic situation associated with precipitation and temperature in Argentina during 2003 and the first quarter of 2004. The period was selected as during that time drought affected at least ten Argentine provinces, causing great agricultural and ranching losses. In order to find the causes of such rainfall deficit the mean and anomaly geopotential height fields at 500 and $1000 \mathrm{hPa}$ were analyzed.

In 2003, rainfall deficit was mainly associated with geopotential anomalies at $500 \mathrm{hPa}$. They show a prevailing flow from the $\mathrm{SW}$, which crosses the country diagonally from $45^{\circ} \mathrm{S}$ approximately to $30^{\circ} \mathrm{S}$. At $1000 \mathrm{hPa}$ the Atlantic Subtropical Anticyclone is weakened, displaced to the north and away from the continent. The Pacific Subtropical Anticyclone is intensified and located to the east with respect to its normal position. The drought in the first quarter of 2004 is mainly due to a ridge at $500 \mathrm{hPa}$ in the center north of the country.

Mean temperature anomalies in the period analyzed are positive throughout the country. High temperatures in 2003 are caused by the input of a N-NE flow from the north of the country, subsidence due to the Pacific Anticyclone shift towards the continent and orography. During the first quarter of 2004 they are generated by a flow from the north and the frequent anticyclonic circulation over the Atlantic to the south of $35^{\circ} \mathrm{S}$.

Keywords: deficit, precipitation, temperature 


\section{INTRODUCCIÓN}

Desde la sequía de 1995 (Alessandro et al. 1996) que afectó a gran parte de la Argentina, no se dieron tantos meses consecutivos deficitarios en gran parte del territorio argentino al norte de la Patagonia como en 2003. Las zonas más deficitarias resultaron ser el Centro oeste de la provincia de La Pampa, sudoeste de Buenos Aires (B. Blanca, Tornquist, Coronel Pringles, etc) y el oeste de Mendoza. Con menor intensidad, se produjo en las provincias de Córdoba, centro oeste de Santiago del Estero, centro oeste de Santa Fe, nordeste de Entre Ríos, norte de Formosa, gran parte de Salta y Chaco.

El primer trimestre de 2004 también resultó deficitario en gran parte del país especialmente en la Mesopotamia (Misiones, Corrientes y Entre Ríos) y el noroeste y centro de la Patagonia (al sur del Río Colorado). En abril en cambio, se produjeron precipitaciones en la mayor parte del país.

El diario La Nación publicó en octubre de 2003, que hasta ese mes, sólo en trigo, soja y girasol, el perjuicio ocasionado en la región afectada fue de 354 millones de dólares. En Mendoza se instaló la sequía más grave de los últimos 40 años, con apenas $70 \mathrm{~mm}$ de agua caídos desde principio de año, que produjo la muerte de 70.000 animales, entre vacunos caprinos y ovinos por la falta de pasturas. En La Pampa esta cifra llegó a 10000 .

Si bien la producción agricolaganadera fue altamente perjudicada, también se vio afectada en un una escala menor la energética. Tal fue el caso de la represa Yaciretá en septiembre y octubre, que superó la dificultad de la disminución del caudal del río Paraná con valores entre los percentiles 25 y 50 por debajo de la serie 1971-2002, a través de la regulación de caudales que efectúa el sistema eléctrico brasileño con sus casi 50 presas situadas aguas arriba de Yacyretá, según lo informado por la Empresa Binacional Yacyretá.

Los meses del período considerado fueron acompañados generalmente por temperaturas medias mayores a las normales. En Junio se produjeron anomalías de temperaturas mayores a $4^{\circ} \mathrm{C}$ en el este de Formosa y Chaco, en el norte de Corrientes y sur de Misiones.

Esta última región incluida en el noreste argentino (NE), conjuntamente con el sur de Brasil, Uruguay y norte de Chile, conforman una región en América del Sur que se halla afectada por los eventos La Niña y El Niño. Estos fenómenos se hallan asociados a una tendencia deficitaria y a excesos de precipitación respectivamente (Aceituno 1988; Diaz et al. 1998; Fontana et al. 1997; Grimm et al. 1996; Grimm et al. 2000; Pisciottano et al. 1994; Rutllant et al. 1991; Rao et al. 1999; Ropelewski et al. 1987) y son diferentes entre sí, tanto en duración como en iniciación e intensidad (Trenberth 1997), causando diferentes impactos en el área afectada.
Alessandro (1998) estudió los aspectos sinópticos de las intensas lluvias producidas durante el período noviembre 1982 a marzo de 1983 (Niño intenso) y Alessandro (2001) las precipitaciones de los mismos meses de los años 97-98 (Niño intenso). En ambos períodos se vió afectado el NE por excesos de precipitación, pero en el segundo con una zona más extendida hacia el sur y hacia el centro del país.

La sequía de 1995 (Alessandro et al. 1995), que afectó la zona húmeda y subhúmeda argentina, se desarrolló bajo condiciones de neutralidad. Esta zona fue nuevamente deficitaria durante la sequía de 1962 ( Malaka et al. 1980), que se produjo bajo la condición de Niña moderada.

De acuerdo a los límites e índices calculados bimensualmente por la National Oceanic and atmosphere Administration (NOAA), el bimestre abril-mayo de 2002 hasta marzo/abril de 2003 corresponden a El Niño. A partir de este último, los valores son neutros, para volver a presentarse el fenómeno aludido anteriormente en septiembre-octubre que se debilita en octubre/noviembre y continúa neutro hasta febrero/ marzo de 2004. Estos períodos donde se presentó El Niño se clasificaron como débiles con un grado de casi fuerte a comienzos de 2003

Uno de los factores más importante en la producción de precipitación es la presencia y profundización de la depresión del noroeste argentino (DNOA). Su intensidad depende de la situación sinóptica y de la época del año, adquiriendo su máximo desarrollo cuando se presenta una masa de aire tropical poco antes del pasaje de una vaguada de onda corta de los oestes. El principal mecanismo de formación y sustentación es la advección horizontal de aire cálido (Lichtenstein 1980).

Varios autores han estudiado la incidencia que tiene el viento de componente este y norte en niveles bajos de la troposfera, sobre la humedad reinante en la Argentina al norte del Río Colorado, y por ende sobre la precipitación. Entre ellos, uno de los primeros trabajos fue el de Fernández et al.(1982) que realizaron un estudio estadístico de la variación vertical y temporal de la velocidad del viento en la región Central y NO de la Argentina. La velocidad del viento mostró un máximo durante los meses de invierno en el tope de la capa límite de dirección predominante del sector norte, ligado al transporte de aire húmedo de regiones tropicales hacia el sur. Inzunza (1992), encontró en Resistencia $\left(27,27^{\circ} \mathrm{S} ; 59,03^{\circ} \mathrm{O}\right)$, el predominio de transporte de humedad desde el norte, en todos los niveles. Nogues-Paegle et al. (1997), estudiaron la oscilación en las condiciones secas y húmedas sobre la región tropical y subtropical al este de los Andes durante el verano, donde jugó un rol dominante la presencia del flujo del sector norte en niveles inferiores. Salio (2000) utilizando un período de tiempo mayor que los que la precedieron, amplió el conocimiento climatológico y sinóptico de la corriente en chorro en capas 
bajas con una discriminación mayor de la dirección del viento del sector NNE.

El propósito de este trabajo es explicar las anomalías de precipitación y evaluar los valores de la temperatura, a través del análisis de las situaciones sinópticas trimestrales y anual en 500 y $1000 \mathrm{hPa}$, así también como la posible vinculación de las primeras con el fenómeno El Niño/ La Niña.

\section{DATOS Y METODOLOGÍA}

El período analizado se extiende desde enero de 2003 hasta marzo de 2004. Se decidió comenzar en enero de 2003 por ser el primer mes del año, lo cual no implica que haya sido el mes del comienzo del período deficitario, y se eligió como último mes al de marzo de 2004, ya que en abril se produjeron precipitaciones en la mayor parte de la Argentina

Se utilizaron para 500 y $1000 \mathrm{hPa}$, las alturas geopotenciales de los reanálisis de 12 UTC del National Center of Enviromental Protection (NCEP) (http://www.cdc. noaa.gov). De este mismo Centro se emplearon también los datos de humedad específica en 1000 y $850 \mathrm{hPa}$ y de viento en 850 y $925 \mathrm{hPa}$. Se eligió este último nivel por estar el viento más alejado del rozamiento superficial que en $1000 \mathrm{hPa}$ y en consecuencia poder tener una mejor información de la dirección de la circulación superficial. Las anomalías de las variables mencionadas, se calcularon respecto al período 1961-1990.

Los valores mensuales de presión, precipitación y temperatura de estaciones argentinas, fueron suministrados por el Servicio Meteorológico Nacional (SMN). Para la obtención de los valores de los desvíos máximos y mínimos de precipitación y temperatura de la República Argentina en el período estudiado, se hizo uso de las estadísticas climatológicas 61-90 y de los Boletines Climatológicos de los años 2003 y 2004, publicados por el SMN.Se adoptó la calificación de eventos El Niño, La Niña o neutros publicada por la NOAA (www.cdc.noaa. gov/people/klaus), realizada a través de los valores del índice multivariado ENSO (MEI), donde los valores positivos se asocian al fenómeno El Niño y los negativos a La Niña .

Se calcularon los porcentajes medios de precipitación acumulada sobre las principales estaciones meteorológicas argentinas, para todos los meses de 2003 y primeros tres de 2004. Estos porcentajes se obtuvieron respecto al valor normal 1961-1990 de precipitación caída en cada una de ellas.

Con el objeto de simplificar las características predominantes de todo el período elegido se calcularon estas cantidades en forma trimestral y anual. Es decir, para cada uno de los cuatro trimestres correspondientes de 2003 (enero, febrero y marzo; abril, mayo y junio; julio, agosto y septiembre; octubre, noviembre y diciembre), para el primero de 2004 y el anual de 2003.
Para conocer la distribución y la disponibilidad de humedad, se procedió a evaluar los campos de humedad específica (q) en 1000 y $850 \mathrm{hPa}$. Dicha disponibilidad se vinculó con el contenido de humedad en $850 \mathrm{hPa}$ por su gran asociación con la formación de precipitación (Showalter, 1953; Gordillo, 1986). Dado que la distribución de la humedad se halla vinculada al campo de viento, se confeccionaron los campos de dirección de viento .

Con el fin de conocer los campos térmicos que acompañaron a este ciclo mayormente deficitario, se hallaron los campos de anomalías de temperatura para los mismos períodos mencionados anteriormente.

Debido al importante papel que desempeña la DNOA sobre la producción de precipitación, es necesario conocer los valores reales de la presión en esta región. Por tal motivo y por una llamativa similitud que se encontró en el noroeste argentino entre situaciones obtenidas por el NCEP en $1000 \mathrm{hPa}$, que respondían a condiciones opuestas de precipitación, se analizó además esta región con los datos de presión reducida al nivel medio del mar del SMN.

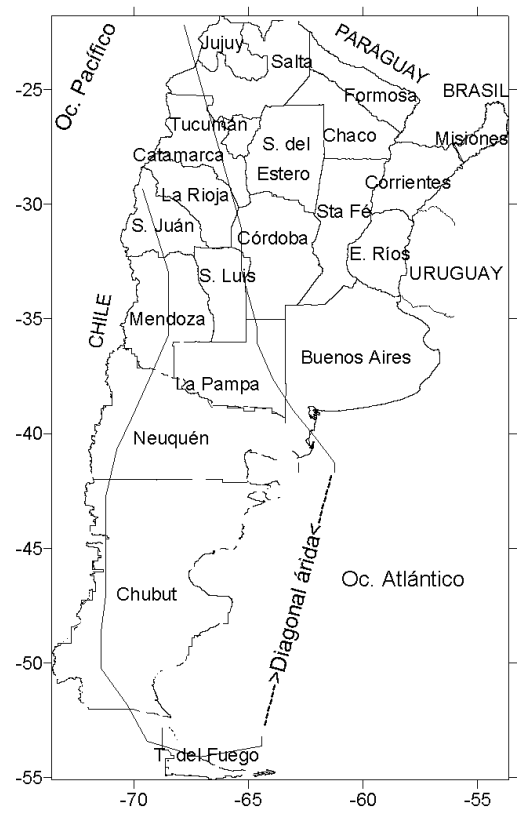

Figura 1 - Mapa político de Argentina

En estos campos medios, en una región determinada de la tropósfera, la presencia de una vaguada, de un centro ciclónico, o de una anomalía negativa con respecto a un período normal, representa el pasaje de perturbaciones con mayor frecuencia o mayor intensidad, con una gran probabilidad de favorecer la producción de precipitaciones (Antico 1998; Ruiz 1998 y 2004). A la inversa cuando aparece una cuña o un anticiclón (o una anomalía positiva) se hace poco posible la producción de la precipitación. Estos conceptos son los que se 
Tabla 1 - Ubicación de las estaciones meteorológicas y precipitaciones medias normales del período 61/90 ( SMN) de las citadas en el trabajo.

\begin{tabular}{|c|c|c|c|c|c|c|c|c|c|c|c|c|c|c|}
\hline \multirow{2}{*}{ Estación } & \multirow{2}{*}{ Lat. } & \multirow[t]{2}{*}{ Long } & \multicolumn{12}{|c|}{ Valores mensuales normales de precipitación } \\
\hline & & & 1 & 2 & 3 & 4 & 5 & 6 & 7 & 8 & 9 & 10 & 11 & 12 \\
\hline BAHIA BLANCA AERO & 38.44 & 62.10 & & & & & & & 30 & & & 70 & & \\
\hline BARILOCHE AERO & 41.09 & 71.10 & & & & & & 141 & 106 & & & & & \\
\hline B. DE IRIGOYEN AERO & 26.15 & 53.39 & & & & & & & & & $\mathrm{~s} / \mathrm{d}$ & & $\mathrm{s} / \mathrm{d}$ & $\mathrm{s} / \mathrm{d}$ \\
\hline BOLIVAR AERO & 36.12 & 61.04 & & & & & & & & & & & & \\
\hline BUENOS AIRES OBS. & 34.35 & 58.29 & 122 & & & & & & & & & & & \\
\hline CATAMARCA AERO & 28.36 & 65.46 & & & & & & & & & & & & \\
\hline CERES AERO & 29.53 & 61.57 & 139 & 134 & & & & & & & & & & \\
\hline CHILECITO AERO & 29.14 & 67.26 & 42 & & & & & & & & & & & \\
\hline C. RIVADAVIA & 45.47 & 67.30 & 11 & & & & & & & & & & & \\
\hline CONCORDIA AERO & 31.18 & 58.01 & & & & 132 & & & & & & & & \\
\hline CORDOBA AERO & 31.19 & 64.13 & & & & & & & & & & & & \\
\hline CORONEL SUAREZ AERO & 37.26 & 61.53 & & & & & & & & & & & & \\
\hline CORRIENTES AERO & 27.27 & 58.46 & & & & & & & & & & & & 121 \\
\hline EL BOLSON AERO & 41.58 & 71.31 & & & & & & 152 & & 94 & 64 & & & \\
\hline ESQUEL AERO & 42.56 & 71.09 & & & & & & & & 36 & & & & \\
\hline EZEIZA AERO & 34.49 & 58.32 & 92 & & & & & & & 58 & & & 109 & \\
\hline FORMOSA AERO & 26.12 & 58.14 & 171 & 121 & & & & & & & & & & \\
\hline GUALEGUAYCHU AERO & 33.00 & 58.37 & & & & & 71 & & 56 & 56 & & & & \\
\hline IGUAZU AERO & 25.44 & 54.28 & 172 & & & & & & & & 137 & 182 & & 135 \\
\hline JACHAL & 30.14 & 68.45 & & & & & & & & & & & & \\
\hline JUNIN AERO & 34.33 & 60.55 & & 105 & & & & & & & & & & \\
\hline LA PLATA AERO & 34.58 & 57.54 & & & & & & & & & & & & \\
\hline LA QUIACA OBS. & 22.06 & 65.36 & 80 & & & & & & & & & & & \\
\hline LA RIOJA AERO & 29.23 & 66.49 & & & & & & & & & & & & \\
\hline LABOULAYE AERO & 34.08 & 63.22 & & & & & & & & & & & 108 & \\
\hline LAS FLORES AERO & 36.02 & 59.08 & 52 & & & & & & & & & & 80 & \\
\hline LAS LOMITAS & 24.42 & 60.35 & 125 & & 112 & & & & & & & & & \\
\hline MAQUINCHAO & 41.15 & 68.44 & - & - & . & - & - & - & - & - & - & - & - & - \\
\hline MARCOS JUAREZ AERO & 32.42 & 62.09 & & & & & & & & & & 95 & 99 & \\
\hline MENDOZA AERO & 32.50 & 68.47 & - & - & & & & - & - & - & - & - & - & - \\
\hline MONTE CASEROS AERO & 30.16 & 57.39 & & & & 138 & & & & & & & & \\
\hline NEUQUEN AERO & 38.57 & 68.08 & - & - & - & - & & - & - & - & - & - & - & - \\
\hline OBERA AERO & 27.29 & 55.08 & & & & & & & 139 & & & & & 131 \\
\hline ORAN AERO & 23.09 & 64.19 & 193 & 167 & 147 & & & & & & & & & \\
\hline PARANA AERO & 31.47 & 60.29 & & & & 102 & & & & & & & & \\
\hline P. DE LOS LIBRES AERO & 29.41 & 57.09 & 135 & 144 & & & 163 & & 83 & 70 & & & & \\
\hline Pte. ROQUE SAENZ PEÑA & 26.49 & 60.27 & 150 & & & & & & & 134 & & & & \\
\hline PERITO MORENO AERO & 46.31 & 71.01 & - & - & - & - & - & - & - & - & - & - & - & - \\
\hline PIGUE AERO & 37.36 & 62.23 & & & & & & & & & & 90 & & \\
\hline PILAR OBS. & 31.40 & 63.53 & & & & & & & & & & & 94 & \\
\hline POSADAS AERO & 27.22 & 55.58 & 156 & 157 & & & 141 & & 131 & & & 178 & 157 & 151 \\
\hline PUERTO DESEADO AE.R. & 47.44 & 65.55 & - & - & - & - & - & - & - & - & - & - & - & - \\
\hline PUNTA INDIO B.A. & 35.22 & 57.17 & 77 & & 123 & & & & & & & & & 87 \\
\hline RECONQUISTA AERO & 29.11 & 59.42 & & & 17 & & & & & & & & & \\
\hline RESISTENCIA AERO & 27.27 & 59.03 & 148 & 171 & & & 97 & & & & & & & \\
\hline RIO CUARTO AERO & 33.07 & 64.14 & 124 & & 111 & & & & & & & & & \\
\hline RIO GALLEGOS AERO & 51.37 & 69.17 & - & - & - & - & - & - & - & - & - & - & - & - \\
\hline RIO GRANDE B.A. & 53.48 & 67.45 & - & - & - & - & - & - & - & - & - & - & - & - \\
\hline SALTA AERO & 24.51 & 65.29 & & 163 & & & & & & & & & & \\
\hline SAN JUAN AERO & 31.34 & 68.25 & - & - & - & - & - & - & - & - & - & - & - & - \\
\hline SAUCE VIEJO AERO & 31.42 & 60.49 & & 132 & & 96 & & & & & & & & \\
\hline TANDIL AERO & 37.14 & 59.15 & - & - & - & - & - & - & - & - & - & - & - & - \\
\hline TARTAGAL AERO & 22.39 & 63.49 & 230 & $\mathrm{~s} / \mathrm{d}$ & $\mathrm{s} / \mathrm{d}$ & & & & & & & & & \\
\hline TINOGASTA & 28.04 & 67.34 & - & - & - & - & - & - & - & - & - & - & - & - \\
\hline
\end{tabular}


aplican aquí, para relacionar los campos medios gepotenciales con ambas variables .

Para facilitar la ubicación de los distintos puntos o regiones que se mencionan en el trabajo, en la Figura 1 se muestra el mapa político de la Argentina y en las columnas 2 y 3 de la Tabla 1, la latitud y longitud de las estaciones meteorológicas citadas.

Dado que el promedio opaca la magnitud de un período más corto deficitario o de exceso, en la Tabla 2 del Anexo se han citado las zonas o estaciones donde se registraron precipitaciones mensuales menores a $25 \mathrm{~mm}$ y entre 150 y $300 \mathrm{~mm}$ y en la Tabla 3 los máximos y mínimos de temperatura. Con el fin de ubicar con mayor facilidad a dichas estaciones, se han marcado con negrita los nombres de las provincias argentinas (Figura 1) a las que pertenecen. Además para evaluar la dimensión de las cantidades de precipitación, en la Tabla 1 se transcriben los valores normales de esta última.

\section{RESULTADOS}

\subsection{Campos Medios}

\subsubsection{De Precipitacion}

En la Figura 2 se muestran los campos trimestrales porcentuales de precipitación para los cuatro trimestres de 2003 (a, b, c y d), primero de 2004 (e) y el anual de 2003 (f). En los correspondientes a 2003, se observan grandes extensiones del territorio con valores menores al valor normal (menores a 100 $\%$ ), así también como una amplia región donde llovió menos o igual a la mitad del mismo. La zona donde llueve menos de la mitad, presenta la mayor extensión, durante el tercer trimestre, resultando nula la precipitación en las estaciones La Rioja y Mendoza Aero. Si bien las zonas varían en cada trimestre, se advierte que las menos afectadas resultaron la del NE argentino y la mitad sur de la Patagonia, como se aprecia en el mapa porcentual anual (Fig. 2 f).Contrariamente en 2004 (Fig. 2e) estas dos útimas presentan valores menores a $100 \%$ y coinciden con el anual de 2003 en el N, NO argentino, norte de la Patagonia y La Pampa. Como se comentó en la introducción esta provincia fue muy afectada durante el período considerado. Por tal motivo, en la Figura 3 se muestra la marcha anual de las anomalías de precipitación de la estación meteorológica pampeana Santa Rosa, respecto a la media de los años 1980-2000. En ella se advierten todos valores negativos.

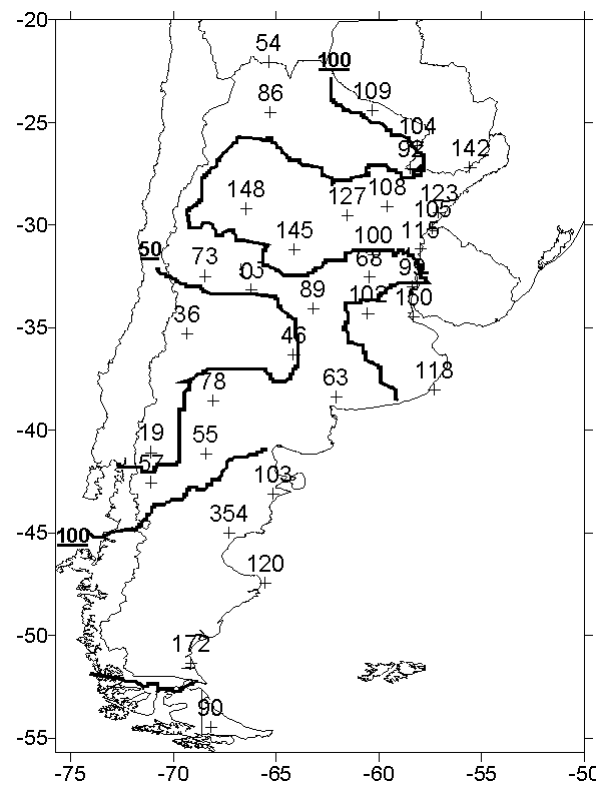

a) $1^{\circ}$ Trimestre de 2003

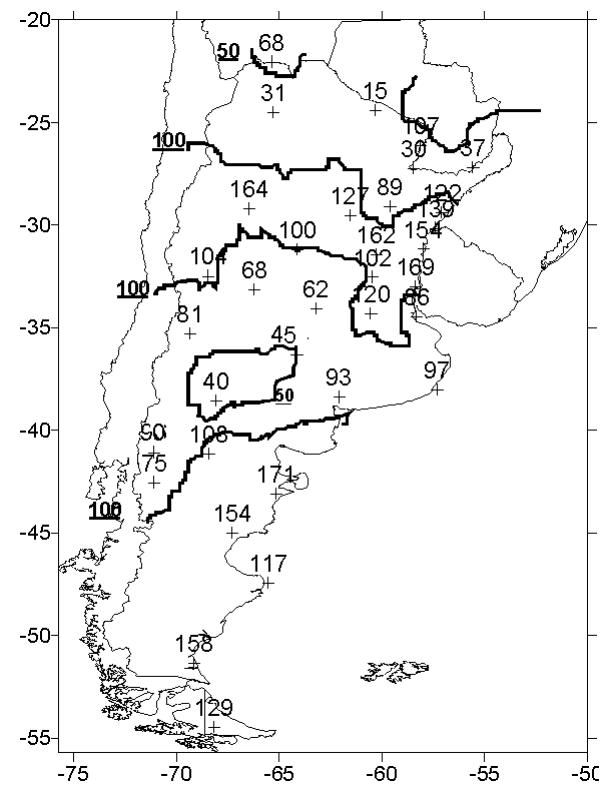

b) $2^{\circ}$ Trimestre de 2003

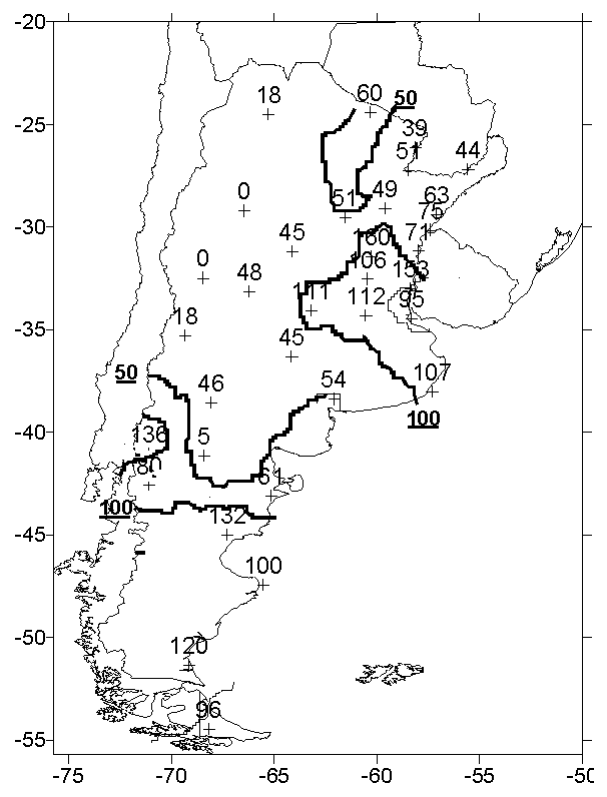

c) $3^{\circ}$ Trimestre de 2003

Figura 2 - Porcentaje de la precipitación acumulada respecto a la normal, de los cuatro trimestres de 2003 (a, b, c y d), primero de 2004 (e) y anual de 2003 (e). 


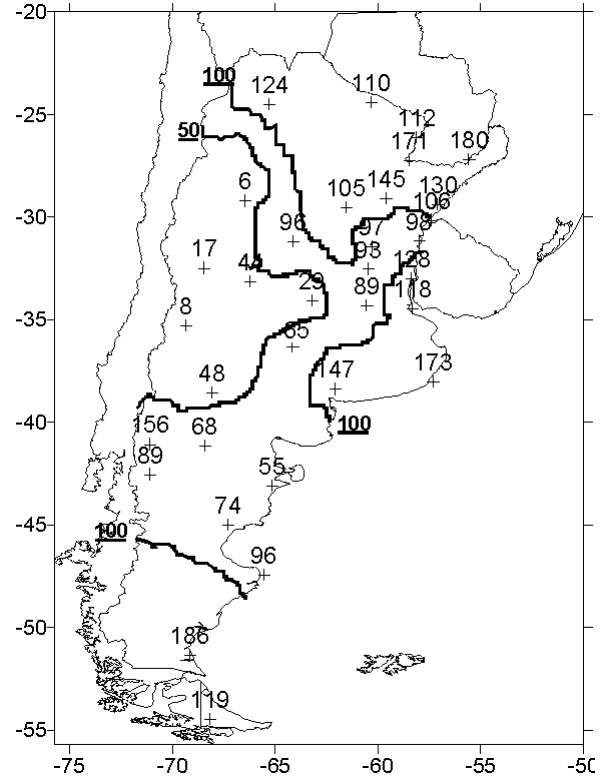

d) $4^{\circ}$ Trimestre de 2003

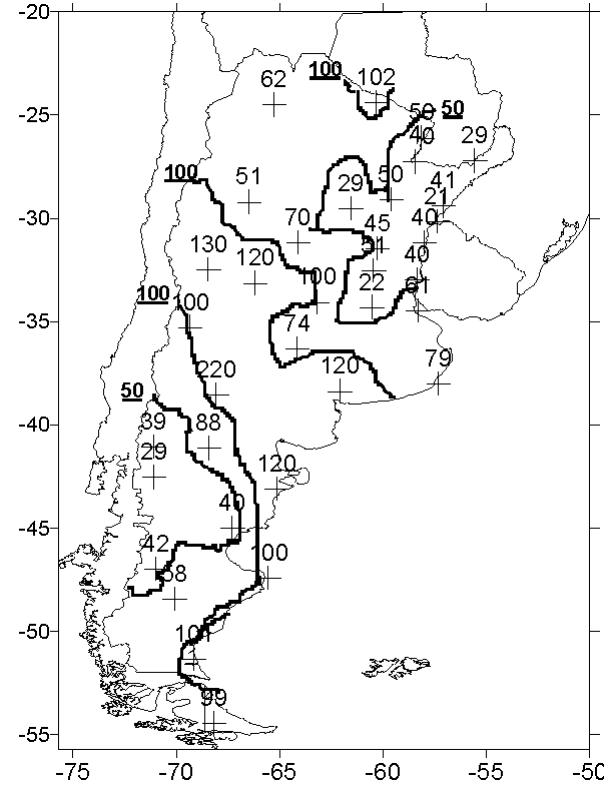

e) $1^{\circ}$ Trimestre de 2004

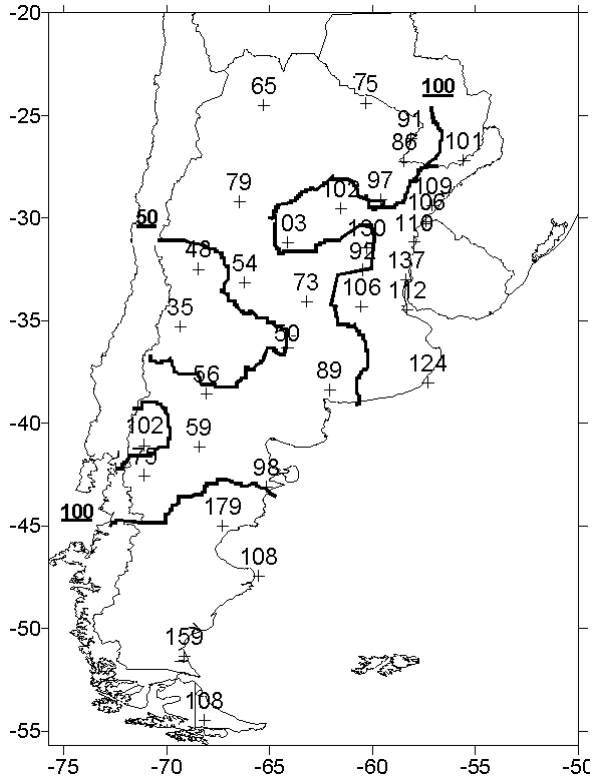

f) Anual de 2003

Figura 2 - continuacion.

\subsubsection{De Anomalías de Humedad Específica}

En la Figura 4 se representan en $850 \mathrm{hPa}$, los campos trimestrales y el anual de las anomalías de humedad específica q; por debajo de cada uno de ellos los campos de las anomalías de la dirección del viento, con el objeto de comprender la distribución de la humedad.

En los campos de viento se representa la dirección anómala de los mismos. Por lo tanto los vectores no representan la dirección real predominante, sino que son la desviación de esta última respecto al valor normal.

Dado que los centros de anomalías con valores significativos en $850 \mathrm{hPa}$, coinciden con la posición que ocupan las anomalías significativas de $1000 \mathrm{hPa}$ y las direcciones de las anomalías de viento en $850 \mathrm{hPa}$ coinciden con las de 925 $\mathrm{hPa}$, por razones de extensión, solo se muestra en $1000 \mathrm{hPa}$ a título de ejemplo el campo correspondiente al primer trimestre de 2004 (Fig. 4g).

Según Fernández (2004) las varianzas de la humedad especifica en $1000 \mathrm{hPa}$ en la Argentina, son mayores en verano y al norte de los $40^{\circ} \mathrm{S}$. Teniendo en cuenta los desvíos típicos calculados por el citado autor en las distintas épocas del año, los valores de las anomalías de q menores a -0.5 $\mathrm{gr} / \mathrm{Kg}$ pertenecientes a los campos representados en la Figura 4 , resultan menores a los mismos. Las mayores anomalías negativas con $\Delta \mathrm{q} \leq-6 \mathrm{gr} / \mathrm{Kg}$ en $1000 \mathrm{hPa} \mathrm{y} \leq-4 \mathrm{gr} / \mathrm{Kg}$ en 850 $\mathrm{hPa}$, se observan en el primer y cuarto trimestre de 2003 y en el primero de 2004.
La ubicación de las mismas corresponden al centro, norte y oeste del país, que es la zona más afectada según lo expuesto en la introducción.

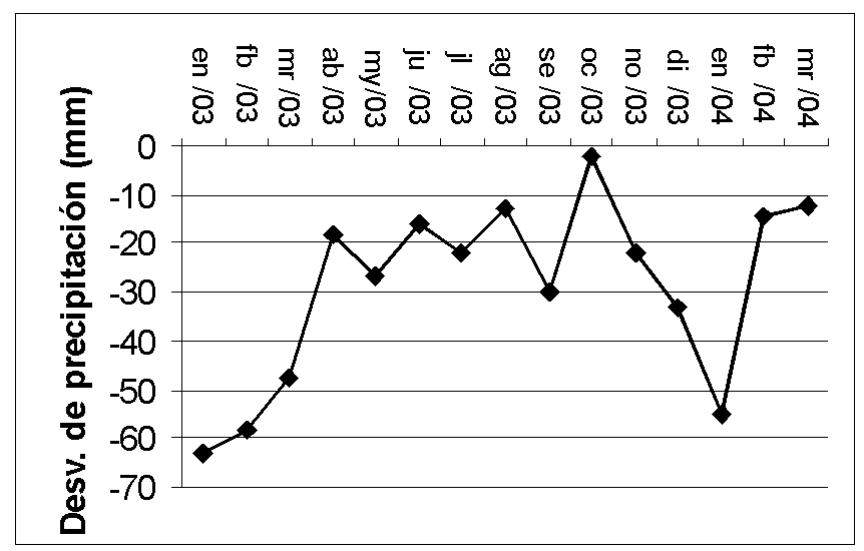

Figura 3 - Marcha anual de anomalías de precipitación en Santa Rosa durante el período analizado. 
Tabla 2 - Valores extremos de precipitación mensual acumulada del período considerado, según lo registrado por el SMN.

\begin{tabular}{|c|c|c|}
\hline mes & Precipitaciones acumuladas mayores a: & Precipitaciones acumuladas menores a: \\
\hline 2003 & $\begin{array}{l}200 \mathrm{~mm} \text { : en Tartagal: } 382.4 \mathrm{~mm} \text { y Orán: } 277.3 \text { (Salta). P. } \\
\text { Roque Sáenz Peña: } 274.0 \mathrm{~mm} \text { (Chaco), Posadas: } 262.3 \mathrm{~mm} \\
\text { (Misiones) y en la costa de Bs As (Punta Indio: } 246.0 \mathrm{~mm} \text { ). }\end{array}$ & $\begin{array}{l}10 \mathrm{~mm} \text { : en el oeste de Catamarca, La Rioja, San Juan y } \\
\text { Mendoza, así como también en gran parte de la Patagonia }\end{array}$ \\
\hline 2003 & $\begin{array}{l}300 \mathrm{~mm} \text { : Ezeiza: } 328.7 \mathrm{~mm} \text { y Ob. Bs As: } 403.6 \mathrm{~mm} \text { ( Bs As) } \\
\text { esta última superó a la máxima cantidad mensual del período } \\
1861-2002 \text {. Sauce Viejo: } 333.6 \mathrm{~mm} \text { (Santa Fé). }\end{array}$ & $10 \mathrm{~mm}$ : en el oeste del territorio y en gran parte de la Patagonia \\
\hline 2003 & $\begin{array}{l}300 \mathrm{~mm} \text { : en Reconquista } 349.7 \mathrm{~mm} \text { y Ceres: } 325.7 \mathrm{~mm} \mathrm{(} \\
\text { Santa Fe). }\end{array}$ & $\begin{array}{l}25 \mathrm{~mm} \text { : en la banda central del país, (entre } 32^{\circ} \mathrm{S} \text { y } 40^{\circ} \mathrm{S} \text { ) . Río } \\
\text { Cuarto (Córdoba), Villa Reynolds (San Luis) y en la costa } \\
\text { rioplatense (Punta Indio) }\end{array}$ \\
\hline $\begin{array}{c}4 \\
2003\end{array}$ & $\begin{array}{l}250 \mathrm{~mm} \text { : en Paraná: } 278.0 \mathrm{~mm} \text { y Concordia:310.4 mm (Entre } \\
\text { Ríos). Monte Caseros: } 256.7 \mathrm{~mm} \text { (Corrientes) y Sauce } \\
\text { Viejo: } 252.0 \mathrm{~mm} \text { (Santa Fe) }\end{array}$ & $\begin{array}{l}25 \mathrm{~mm} \text { en casi toda la diagonal árida. Las Lomitas con } 2.3 \mathrm{~mm} \\
\text { (Formosa), fué inferior al mínimo valor mensual de abril del } \\
\text { período 1961-1990 }\end{array}$ \\
\hline 2003 & $\begin{array}{l}150 \mathrm{~mm} \text { : en Gualeguaychú: } 179.3 \mathrm{~mm} \text { y Concordia: } 170 \mathrm{~mm} \\
\text { (Entre Ríos). Paso de los Libres: } 167.5 \mathrm{~mm} \text { (Corrientes). }\end{array}$ & $\begin{array}{l}\text { I0 mm: en gran parte de Mendoza, S. Juan, S. Luis, oeste de } \\
\text { Córdoba y La Pampa, norte de la Patagonia y extremo NE } \\
\text { del país. Posadas con } 0.5 \mathrm{~mm} \text { (Misiones) fué el menor valor del } \\
\text { período 1902-2002. Resistencia con } 2.4 \mathrm{~mm} \text { (Chaco) resultó } \\
\text { inferior al mínimo del período 1966-2002. }\end{array}$ \\
\hline $\begin{array}{c}6 \\
2003 \\
\end{array}$ & $\begin{array}{l}200 \mathrm{~mm} \text { : en Bariloche: } 289.4 \mathrm{~mm} \text { y en El Bolsón: } 263.0 \mathrm{~mm} \text { ( } \\
\text { Río Negro). }\end{array}$ & $\begin{array}{l}10 \mathrm{~mm} \text { : en la mayor parte del territorio al norte de } 37^{\circ} \mathrm{S} \text {, a } \\
\text { excepción del extremo NE del país }\end{array}$ \\
\hline $\begin{array}{r}7 \\
2003 \\
\end{array}$ & $100 \mathrm{~mm}$ : en Bariloche: $113.9 \mathrm{~mm}$ ( Río Negro). & $\begin{array}{l}10 \mathrm{~mm} \text { : en la mayor parte del territorio. Bahía Blanca con } 0.4 \\
\text { mm (Bs. As.) resultó menor a la mínima precipitación del } \\
\text { período 1961- }\end{array}$ \\
\hline 2003 & $\begin{array}{l}100 \mathrm{~mm} \text { : en Gualeguaychú: } 129.3 \mathrm{~mm} \text { (E. Ríos ) y Paso de } \\
\text { los Libres: } 114.2 \mathrm{~mm} \text { (Corrientes). El Bolsón: } 113.0 \mathrm{~mm} \text { (Río } \\
\text { Negro) y Esquel : } 107.2 \mathrm{~mm} \text { (Chubut). }\end{array}$ & $10 \mathrm{~mm}$ : en casi toda la diagonal árida. \\
\hline 2003 & 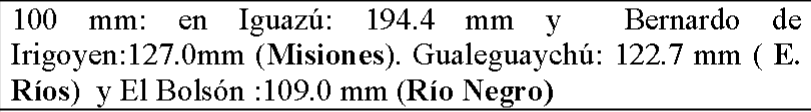 & $10 \mathrm{~mm}$ : en casi toda la diagonal árida. \\
\hline 2003 & $\begin{array}{l}150 \mathrm{~mm} \text { : en Posadas: } 270.0 \mathrm{~mm} \text { y Bernardo de Irigoyen: } 265.0 \\
\text { mm (Misiones). Pigüé: } 181.0 \mathrm{~mm} \text { y Bahía Blanca: } 157.2 \mathrm{~mm} \\
\text { (Bs. As.) }\end{array}$ & $\begin{array}{l}10 \mathrm{~mm} \text { : en casi toda la diagonal árida. Marcos Juárez: } 12.1 \mathrm{~mm} \\
\text { (Córdoba) y en Villa Reynolds: } 1.9 \mathrm{~mm} \text { (S. Luis) fueron más } \\
\text { bajos que los mínimos mensuales de octubre registrados en el } \\
\text { período 1961- }\end{array}$ \\
\hline 2003 & $\begin{array}{l}150 \mathrm{~mm} \text { : en Bernardo de Irigoyen: } 277.3 \mathrm{~mm} \text { y Posadas: } 239.2 \\
\text { mm (Misiones). Las Flores: } 232.0 \mathrm{~mm} \text { en y Buenos Aires } \\
\text { Observatorio: } 230 \mathrm{~mm} \text { ( Bs As). }\end{array}$ & $\begin{array}{l}10 \mathrm{~mm} \text { : en toda la diagonal árida. Villa Reynolds: } 11.0 \mathrm{~mm} \text { y } \mathrm{S} \text {. } \\
\text { Luis:11.2 mm (San Luis). Marcos Juárez }: 18.2 \mathrm{~mm} \text {, Río Cuarto } \\
: 19.0 \mathrm{~mm} \text {, Pilar :38.6 mm y Laboulaye: } 40.8 \mathrm{~mm} \text { (Córdoba) } \\
\text { fueron más bajos que los mínimos registrados en el período } \\
\text { 1961-1990. }\end{array}$ \\
\hline 2003 & $\begin{array}{l}300 \mathrm{~mm} \text { : en Bernardo de Irigoyen: } 515.0 \mathrm{~mm} \text {, Iguazú: } 508.0 \\
\text { mm y Posadas: } 329.1 \mathrm{~mm} \text { (Misiones). Corrientes: } 351.4 \mathrm{~mm} \text { y } \\
\text { Oberá: } 318.0 \mathrm{~mm} \text { (Corrientes). Todos estos valores superiores } \\
\text { a los máximos del período } 1961-1990\end{array}$ & $10 \mathrm{~mm}$ : en toda la diagonal árida \\
\hline 2004 & $\begin{array}{l}200 \mathrm{~mm} \text { : en Obs. Bs As :268.4 mm y Punta Indio: } 238.0 \mathrm{~mm} \\
\text { (Bs As). Este último superó el máximo mensual del mes de } \\
\text { enero de los últimos } 43 \text { años. Algo similar ocurrió en La } \\
\text { Quiaca: } 165 \mathrm{~mm} \text { (Jujuy), valor que superó al máximo total } \\
\text { mensual de enero para el período 1961-2003. }\end{array}$ & $\begin{array}{l}10 \mathrm{~mm} \text { en el borde occidental del territorio y en la mayor parte } \\
\text { de la Patagonia. Paso de los Libres: } 11.1 \mathrm{~mm} \text { (Corrientes), } \\
\text { Ceres: } 13.0 \mathrm{~mm} \text { (Santa Fé), Formosa: } 12.8 \mathrm{~mm} \text { (Formosa) y } \\
\text { (Resistencia: } 35.8 \mathrm{~mm} \text { (Chaco) fueron inferiores a los } \\
\text { mensualess de enero del período } 1961-2003 \text { y el de Villa } \\
\text { Dolores: } 38.3 \mathrm{~mm} \text { (Córdoba) inferior al mínimo histórico de } \\
\text { enero. }\end{array}$ \\
\hline 2004 & $\begin{array}{l}150 \mathrm{~mm} \text { : en Orán } 185.2 \mathrm{~mm} \text {, Salta: } 174.5 \mathrm{~mm} \text { y Tartagal: } \\
171.9 \mathrm{~mm} \text { (Salta). }\end{array}$ & $\begin{array}{l}10 \mathrm{~mm} \text { : en el borde occidental del territorio y en el centro de la } \\
\text { Patagonia. Posadas: } 47.2 \mathrm{~mm} \text { (Misiones), Resistencia: } 44.6 \\
\text { mm (Chaco), Rafaela }: 29.1 \mathrm{~mm} \text { (Santa Fé) y Junin: } 20.0 \mathrm{~mm} \text { ( } \\
\text { Bs. As.) resultaron inferiores a los mínimos del periodo } 1961- \\
1990 \text {. }\end{array}$ \\
\hline $\begin{array}{l}3 \\
2004 \\
\end{array}$ & $\begin{array}{l}150 \mathrm{~mm} \text { : en Tartagal: } 235.9 \mathrm{~mm} \text { y Orán }: 173.6 \mathrm{~mm} \text { (Salta). } \\
\text { Las Lomitas : } 197.2 \mathrm{~mm} \text { (Formosa) y en Viedma: } 156.0 \mathrm{~mm} \\
\text { (Río Negro) valor superior al máximo del periodo } 1971-2003 \text {. }\end{array}$ & $\begin{array}{l}10 \mathrm{~mm} \text { : en el borde occidental del territorio y en el centro de la } \\
\text { Patagonia }\end{array}$ \\
\hline
\end{tabular}


Tabla 3 - Valores extremos de anomalías de temperatura del período considerado, según lo registrado por el SMN.

\begin{tabular}{|c|c|c|}
\hline mes & Máximos desvíos positivos de temperatura en: & Mínimos desvíos negativos de temperatura en: \\
\hline $\begin{array}{c}1 \\
2003\end{array}$ & $\begin{array}{l}\text { Chilecito: } 2.5^{\circ} \mathrm{C} \text { (La Rioja), Tinogasta: } 2.3^{\circ} \mathrm{C} \text { (Catamarca), } \\
\text { Mendoza: } 1.8^{\circ} \mathrm{C} \text { (Mendoza) y S.Jjuan: } 1.5^{\circ} \mathrm{C} \text { (San Juan). }\end{array}$ & Río Grande: $-1^{\circ} \mathrm{C}$ (Tierra del Fuego) \\
\hline $\begin{array}{c}2 \\
2003\end{array}$ & $\begin{array}{l}\text { La Quiaca: } 2.1^{\circ} \mathrm{C} \text { (Jujuy), Catamarca: } 2.0^{\circ} \mathrm{C} \text { (Catamarca) y } \\
\text { Chilecito: } 2.0^{\circ} \mathrm{C} \text { ( La Rioja). }\end{array}$ & Bolivar:-1. $0^{\circ} \mathrm{C}$ (Bs As) \\
\hline 2003 & $\begin{array}{l}\text { Coronel Suárez : } 2.7^{\circ} \mathrm{C} \text { y Bahía Blanca: } 2.5^{\circ} \mathrm{C} \text { (Bs As). } \\
\text { Bariloche: } 2.5^{\circ} \mathrm{C} \text {. Maquinchao: } 2.1^{\circ} \mathrm{C} \text {, (Neuquen): } 1.9^{\circ} \mathrm{C} \\
\text { (Patagonia). Mendoza: } 1.8^{\circ} \mathrm{C} \text { (Men doza) }\end{array}$ & $\begin{array}{l}\text { NE del país y en el sudeste de Santa Cruz. Los } \\
\text { mínimos fueron leves y no alcanzaron a }-1^{\circ} \mathrm{C} \text {. }\end{array}$ \\
\hline $\begin{array}{c}4 \\
2003 \\
\end{array}$ & $\begin{array}{l}\text { La Quiaca: } 1.5^{\circ} \mathrm{C} \text { (Jujuy). Río Grande: } 1.2^{\circ} \mathrm{C} \text { y en Ushuaia: } \\
1.1^{\circ} \mathrm{C} \text { ( Tierra del Fuego) }\end{array}$ & $\begin{array}{l}\text { Concordia:- } 1.4^{\circ} \mathrm{C} \text { (E. Ríos). Tandil, Tres Arroyos y } \\
\text { Bolívar : }-1.2^{\circ} \mathrm{C}(\mathrm{Bs} \text { As). }\end{array}$ \\
\hline $\begin{array}{c}5 \\
2003 \\
\end{array}$ & $\begin{array}{l}\text { La Quiaca: } 2.5^{\circ} \mathrm{C} \text { (Jujuy). Ushuaia: } 2.2^{\circ} \mathrm{C} \text { ( Tierra del } \\
\text { Fuego). }\end{array}$ & P. Roque Sáenz Peña: $-1^{\circ} \mathrm{C}$ (Chaco) \\
\hline 2003 & $\begin{array}{l}\text { Las Lomitas: } 5^{\circ} \mathrm{C} \text { (Formosa). Resistencia: } 4.8^{\circ} \mathrm{C} \text { y P. Roque } \\
\text { Sáez Peña: } 4^{\circ} \mathrm{C} \text { (Chaco), Corrientes: } 4.1{ }^{\circ} \mathrm{C} \text { (Corrientes) y } \\
\text { Posadas: } 4.1^{\circ} \mathrm{C} \text { (Misiones). }\end{array}$ & $\begin{array}{l}\text { SE del país donde tuvieron lugar leves desvíos } \\
\text { negativos. }\end{array}$ \\
\hline $\begin{array}{c}7 \\
7 \\
2003 \\
\end{array}$ & Perito Moreno: $3.4^{\circ} \mathrm{C}, \mathrm{y}$ Río Gallegos: $3.1^{\circ} \mathrm{C}$ (Santa Cruz). & $\begin{array}{l}\text { En el centro-este de país tuvieron lugar leves desvíos } \\
\text { negativos. }\end{array}$ \\
\hline $\begin{array}{c}8 \\
8 \\
2003\end{array}$ & $\begin{array}{l}\text { Puerto Deseado: } 1.5^{\circ} \mathrm{C} \text { (Santa Cruz). Ushuaia: } 1.6 \text { (Tierra } \\
\text { del Fuego) }\end{array}$ & $\begin{array}{l}\left.\text { Chaco (P. Roque Sáenz Peña: }-2.5^{\circ} \mathrm{C}\right) \text { y Santa Fé ( } \\
\text { Ceres: }-2.1^{\circ} \mathrm{C} \text { ). }\end{array}$ \\
\hline 2003 & $\begin{array}{l}\text { Jachal: } 2.6^{\circ} \mathrm{C} \text { (San Juan), Chilecito: } 2.5^{\circ} \mathrm{C} \text { (La Rioja) y } \\
\text { Catamarca: } 2.4^{\circ} \mathrm{C} \text { (Catamarca). }\end{array}$ & $\begin{array}{l}\text { El este de Bs As y la franja costera de la provincia de } \\
\text { Santa Cruz se produjeron leves desvíos negativos que } \\
\text { no alcanzaron a }-1.0^{\circ} \mathrm{C}\end{array}$ \\
\hline $\begin{array}{c}10 \\
2003 \\
\end{array}$ & $\begin{array}{l}\text { S. Luis: } 3.9^{\circ} \mathrm{C} \text { y Villa Reynolds: } 3.2^{\circ} \mathrm{C} \text {, (San Luis) .Mendoza } \\
: 3.2^{\circ} \mathrm{C} \text { (Mendoza) y Tinogasta }: 3.2^{\circ} \mathrm{C} \text { (Catamarca). }\end{array}$ & $\begin{array}{l}\text { El sur de la región patagónica anomalías negativas } \\
\text { leves. Río Gallegos: }-1^{\circ} \mathrm{C} \text { (Santa Cruz). }\end{array}$ \\
\hline $\begin{array}{c}11 \\
2003\end{array}$ & $\begin{array}{l}\text { Chilecito: } 3.6^{\circ} \mathrm{C} \text { (La Rioja), Jáchal: } 3.0^{\circ} \mathrm{C} \text { (San Juan) y Villa } \\
\text { Reynolds: } 2.9^{\circ} \mathrm{C} \text { ( S. Luis). }\end{array}$ & $\begin{array}{l}\text { E1 extremo sur del país: Ushuaia: }-1.7^{\circ} \mathrm{C} \text { (Tierra del } \\
\text { Fuego) y Río Gallegos:-1.7 (Sant a Cruz ). }\end{array}$ \\
\hline 2003 & $\begin{array}{l}\text { Chilecito: } 1.9 \quad \mathrm{C}^{\circ} \quad(\mathrm{La} \text { Rioja }) \\
\text { Catam arca). }\end{array}$ & $\begin{array}{l}\text { Oberá:- } 2.9^{\circ} \mathrm{C} \text { y Paso de los Libres- } 2.4^{\circ} \mathrm{C}(\text { (Corrientes), } \\
\text { Concordia: }-2.2^{\circ} \mathrm{C} \text { (E. Ríos), Villa de María:- } 2.4^{\circ} \mathrm{C} \\
\text { (Córdoba) y en el centro-oeste de la Patagonia } \\
\text { (Maquinchao: }-2.6^{\circ} \mathrm{C} \text {, Bariloche: }-2.4^{\circ} \mathrm{C} \text { y Paso de } \\
\text { Indios: }-2.3^{\circ} \mathrm{C} \text { ). }\end{array}$ \\
\hline $\begin{array}{c}1 \\
2004 \\
\end{array}$ & $\begin{array}{l}\text { Villa Dolores: } 3.1^{\circ} \mathrm{C} \text { (Córdoba), Jáchal: } 2.8^{\circ} \mathrm{C} \text { ( San Juan), } \\
\text { Chilecito: } 2.6^{\circ} \mathrm{C} \text { (La Rioja), y en la región Patagónica } \\
\text { (Neuquén: } 2.6^{\circ} \mathrm{C} \text {, Viedma y Esquel } 2.4^{\circ} \mathrm{C} \text { ) }\end{array}$ & \\
\hline $\begin{array}{c}2 \\
2004 \\
\end{array}$ & El noroeste de Patagonia (Esquel: $3.4^{\circ}$, Bariloche: $2.7^{\circ} \mathrm{C}$ ). & $\begin{array}{l}\text { Laboulage }-2.0^{\circ} \mathrm{C} \text { y Marcos Juárez: } 1.8^{\circ} \mathrm{C} \text { (Córdoba). } \\
\text { La Plata: }-2.0^{\circ} \mathrm{C} \text { y Punta Indio: }-1.7^{\circ} \mathrm{C} \text { ( Bs As). }\end{array}$ \\
\hline $\begin{array}{c}3 \\
2004 \\
\end{array}$ & $\begin{array}{l}\text { Coronel Suárez:: } 3.9^{\circ} \mathrm{C} \text {, Las Flores: } 3.8^{\circ} \mathrm{C} \text { y Bahía Blanca: } \\
3.3^{\circ} \mathrm{C} \text { ( Bs As). }\end{array}$ & \\
\hline
\end{tabular}

\section{3-1.3. De Anomalías de Temperatura}

El análisis mensual de las anomalías de temperatura, que por utilizar menor espacio no se muestra, determina el predominio de las de signo positivo. El único mes de 2003 que presentó valores de anomalías negativas en casi todo el país correspondió al mes de diciembre, y de los meses considerados de 2004 solo febrero al norte de los $40^{\circ} \mathrm{S}$. En la Figura 5, se representan los cuatro campos trimestrales correspondientes a 2003 y el primero de 2004, así también como el anual de 2003. Casi todos ellos con valores de anomalías de temperatura positivas, si bien ellas no superaron los valores de los desvíos típicos.
En el año 2003, se presentaron anomalías menores a $1^{\circ} \mathrm{C}$ en la Patagonia y en el este del país en el primer y segundo trimestre y en una de estas dos regiones en el segundo y en el tercero respectivamente. Distribución de valores que se ven reflejados en el campo anual, donde se observan anomalías comprendidas entre 1 y $1.7^{\circ} \mathrm{C}$ fuera de las dos región la regiones aludidas. En 2004 las anomalías se incrementan (entre 1 y $2.5^{\circ} \mathrm{C}$ ) y ocupan una región más extensa que incluye a la Patagonia.

En la Tabla 1 del Anexo se han transcrito los máximos desvíos positivos y negativos de los meses considerados, con el objeto de cuantificar la magnitud de los mismos. 

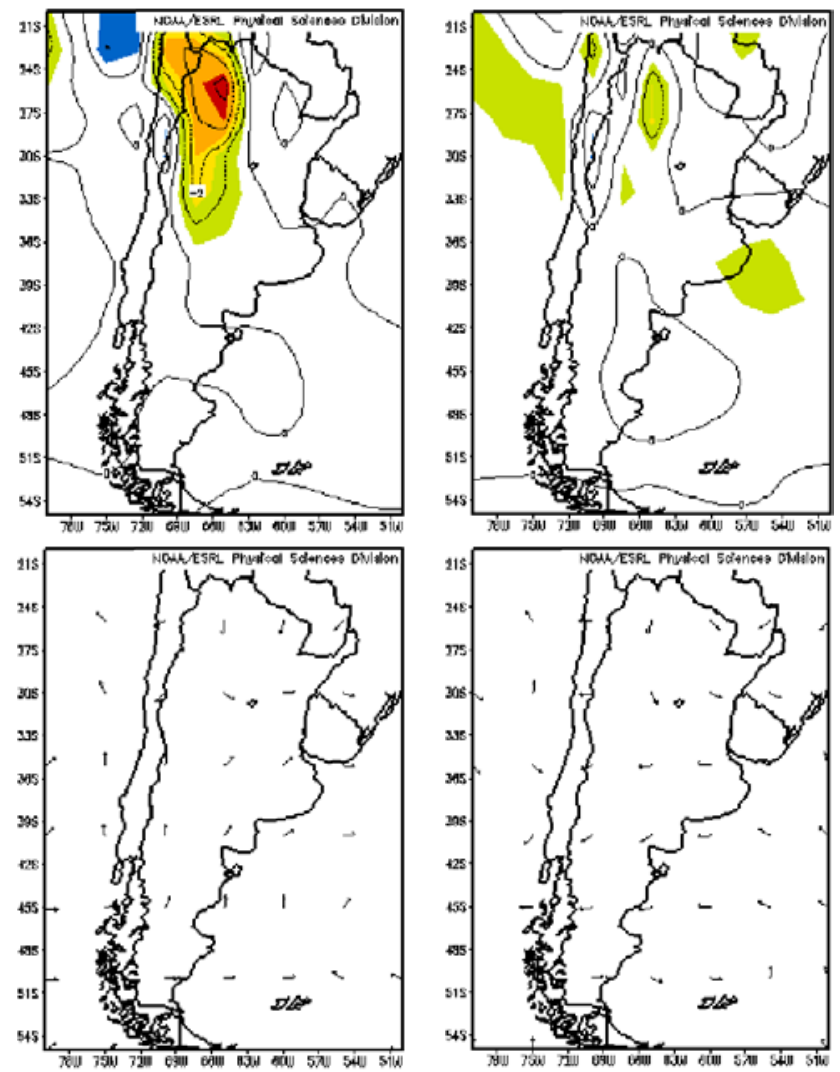

a) $1^{\circ}$ Trimestre 2003
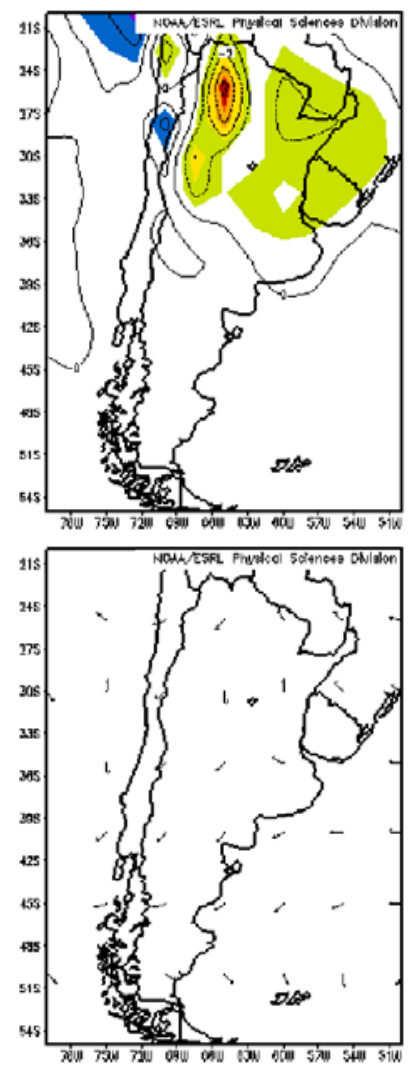

e) $1^{\circ}$ Trimestre 2004

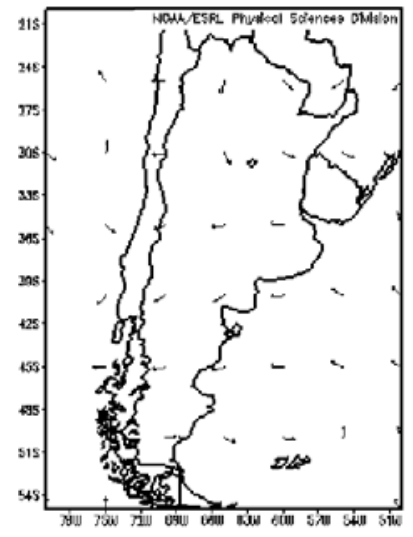

b) $2^{\circ}$ Trimestre de 2003
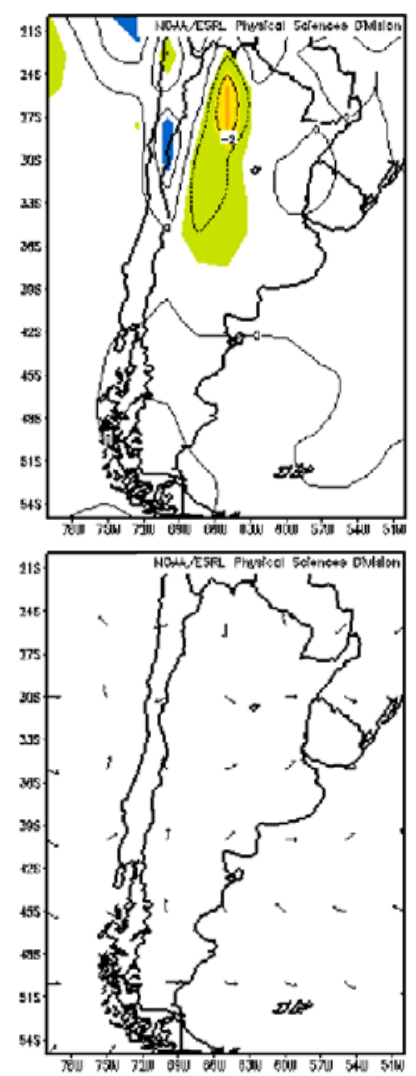

f) Anual 2003
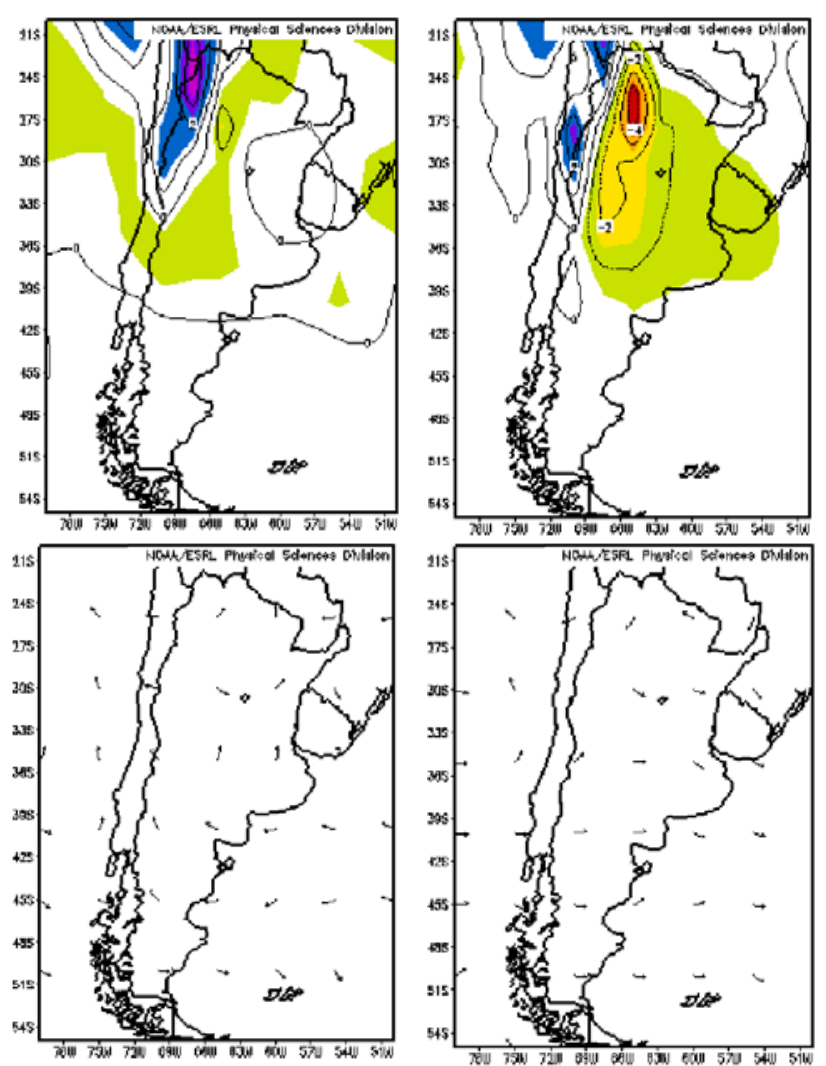

c) $3^{\circ}$ Trimestre de 2003

d) $4^{\circ}$ Trimestre de 2003
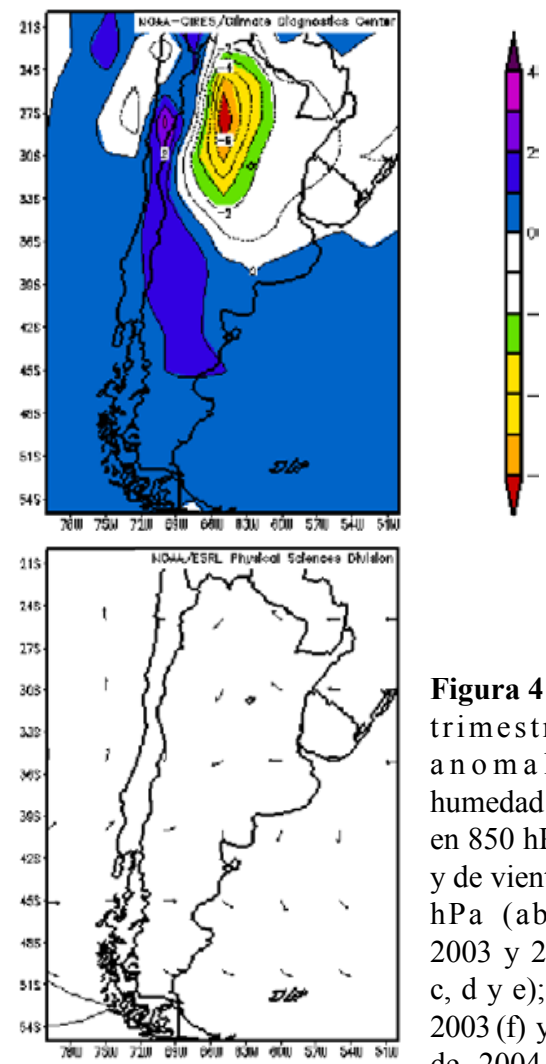

g) $1^{\circ}$ Trimestre 2004 en $1000 \mathrm{hPa}$
Figura 4 - Campos trimestrales de anomalías de humedad específica en $850 \mathrm{hPa}$ (arriba) y de vientos en 925 hPa (abajo) de: 2003 y 2004 (a, b, c, d y e); anual de 2003 (f) y trimestral de $2004(\mathrm{~g})$ pero en $1000 \mathrm{hPa}$. 


\subsubsection{De los campos de presíon en el noroeste Argentino}

Se confeccionan todos los campos mensuales de anomalías de presión reducidas al nivel medio del mar (no se muestran) con los datos proporcionados por el SMN. Las anomalías se presentan positivas en el extremo del NO, en enero, marzo, abril, mayo, julio y agosto. En éstos dos últimos meses este signo no solo se presenta en la aludida región sino en todo el país, llegando en agosto a producirse anomalías positivas significativas al 15\% de una distribución normal. En el resto de los meses el valor de las anomalías se presentan mayormente negativas en el NO.

Los campos calculados se comparan con los campos medios de anomalías de alturas geopotenciales en $1000 \mathrm{hPa}$ obtenidos del modelo del NCEP. Estos últimos muestran en el noroeste del país, anomalías de signo positivo para los meses que poseen anomalías negativas de presión. Como ejemplo se muestran los campos de febrero y junio (Fig. 6) de 2003. a) $1^{\circ}$ Trimestre de 2003

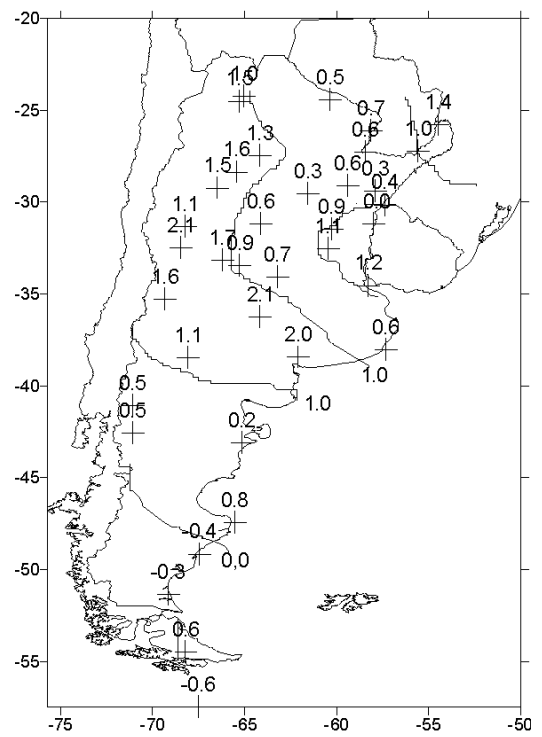

d) $4^{\circ}$ Trimestre de 2003

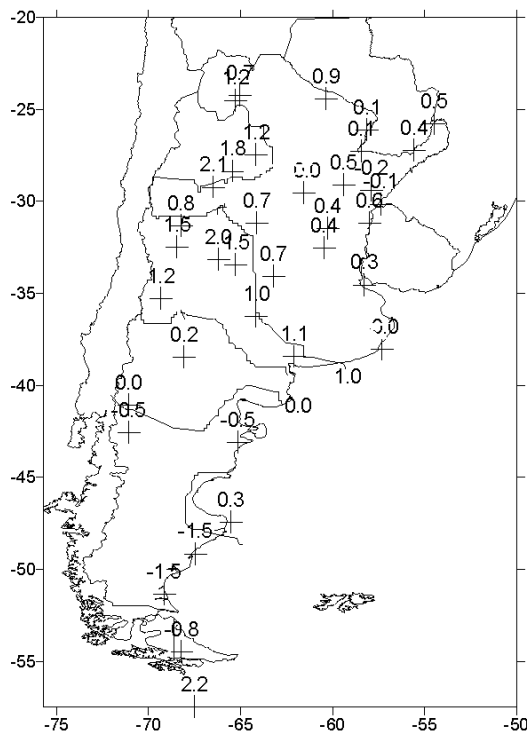

b) $2^{\circ}$ Trimestre de 2003

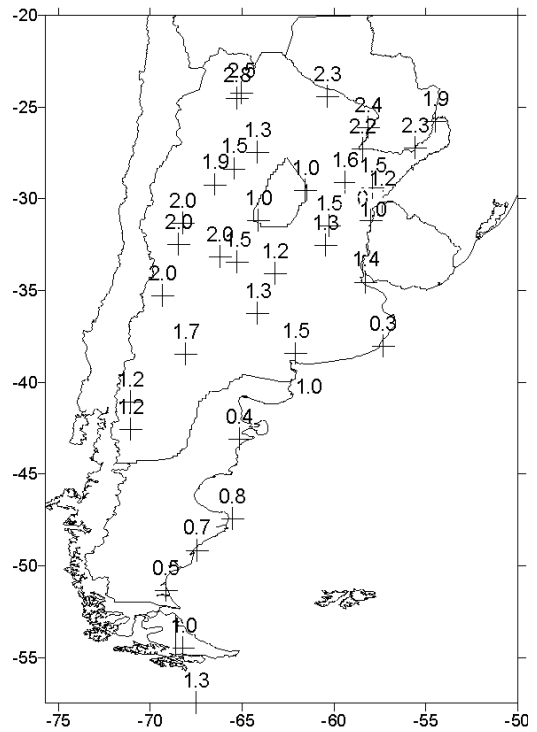

e) Anual de 2003

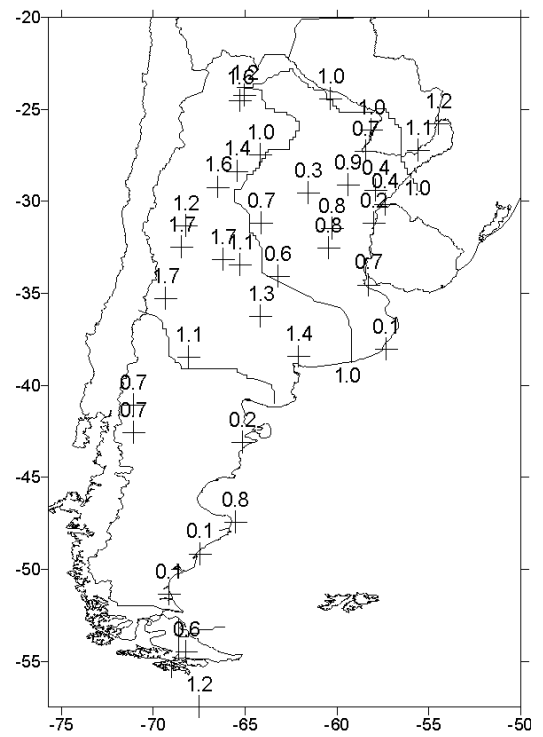

c) $3^{\circ}$ Trimestre de 2003

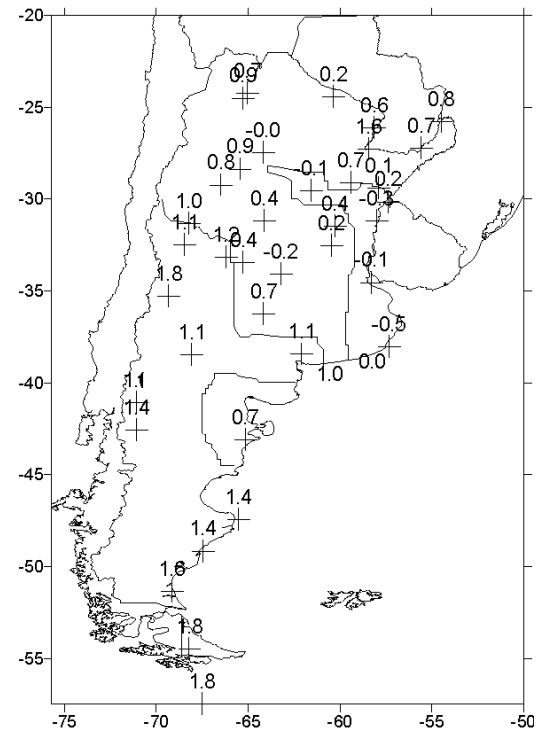

f) Anual de 2004

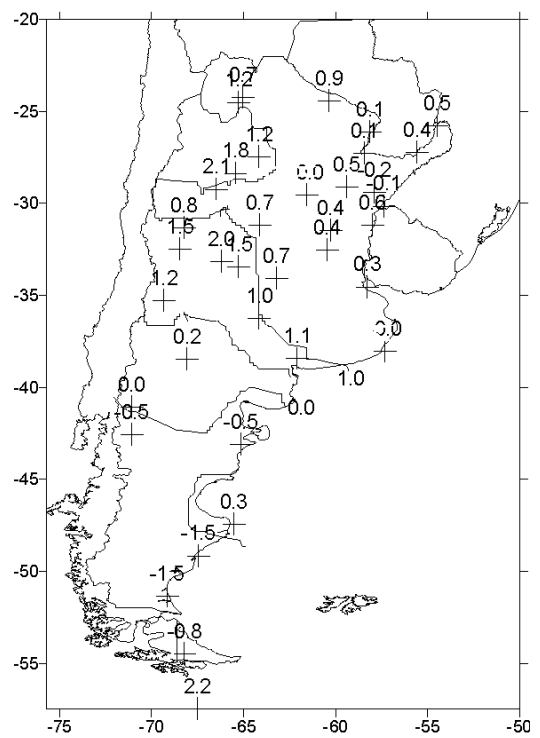

Figura 5 - Anomalías trimestrales de temperatura de los cuatro trimestres de 2003 (a, b, c y d), primero de 2004 (e) y anual de 2003 (e). 


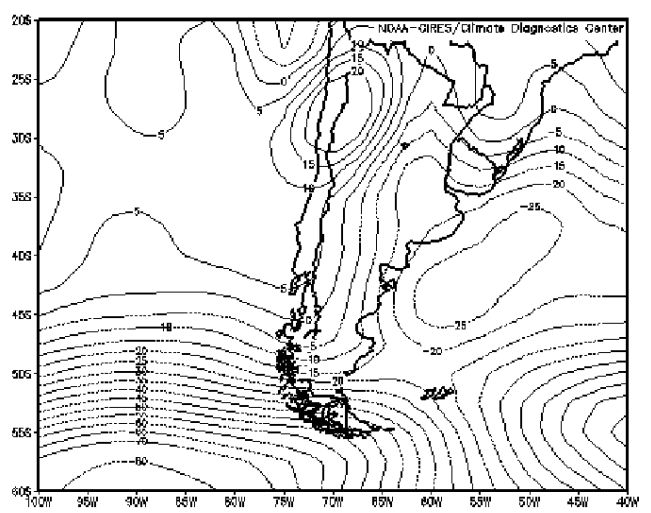

a) Febrero 2003

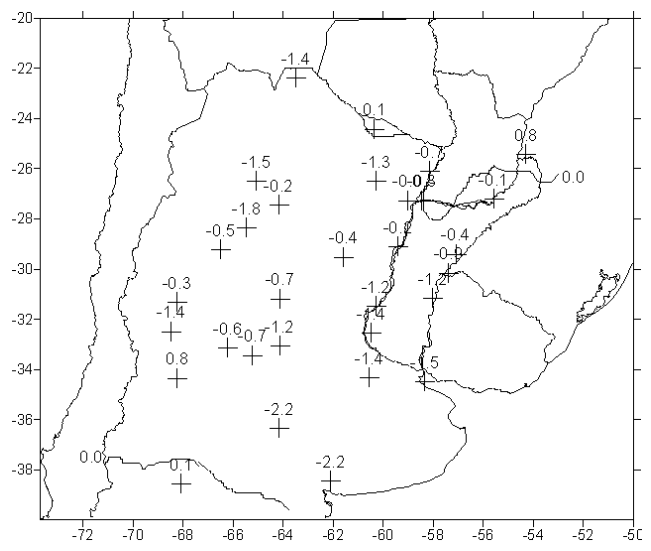

c) Febrero 2003

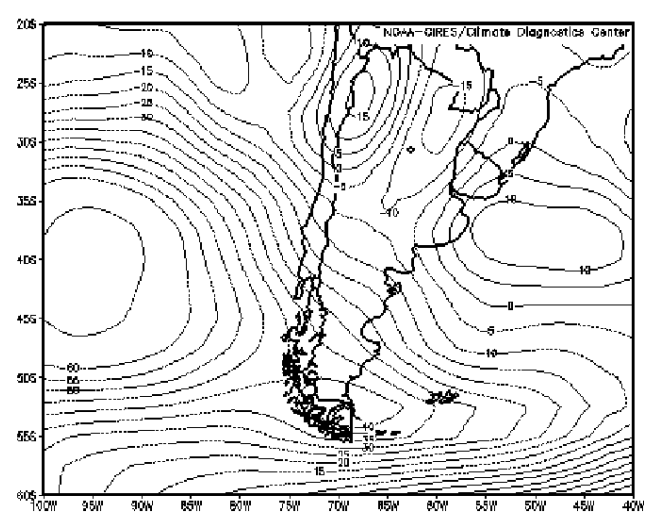

b) Junio 2003

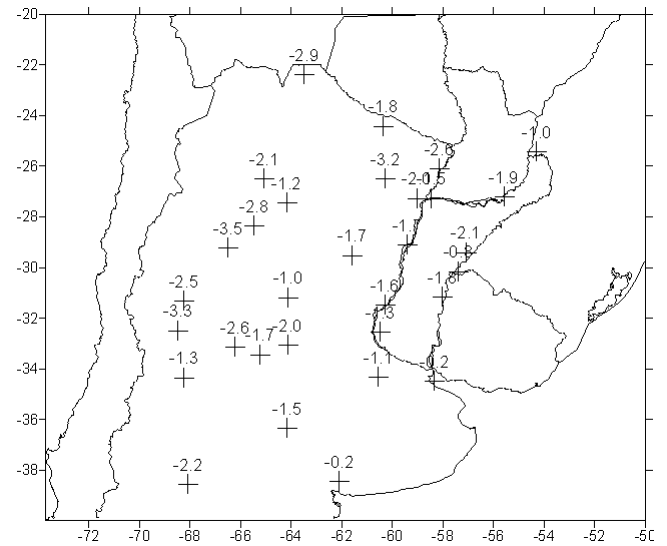

d) Junio 2003

Figura 6 - Campos de anomalías mensuales de alturas geopotenciales de $1000 \mathrm{hPa}$. Campos de anomalías mensuales de presión c) y d).

El máximo positivo espúreo representado por las salidas gráficas del NCEP produce un desplazamiento de la DNOA, con distorsiones sobre las variables meteorológicas esperadas en los meses de febrero, junio, septiembre, octubre, noviembre $\mathrm{y}$ diciembre.

\subsubsection{De alturas geopotenciales}

El campo de anomalías hemisféricas de altura geopotencial de $500 \mathrm{hPa}$ (Fig. 7), presenta dos anomalías positivas en el Océano Pacífico. La mayor se halla en el promedio anual en $43^{\circ} \mathrm{S}$ y $145^{\circ} \mathrm{O}$ y con una ubicación casi constante en el oeste del Océano. El otro centro positivo se presenta al este del centro anterior y al oeste de la Argentina, es más débil y variable en su posición pero afecta a Sudamérica en todos los trimestres de 2003.

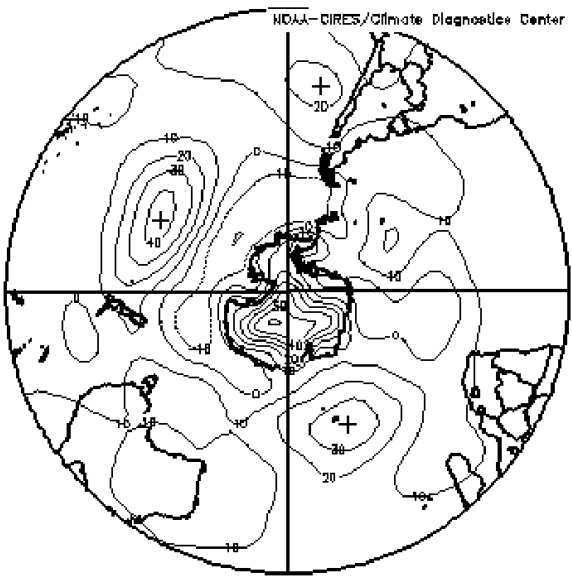

Figura 7 - Campo hemisférico de anomalías anuales de alturas geopotenciales en $500 \mathrm{hPa}$ en 2003. NOAA. 


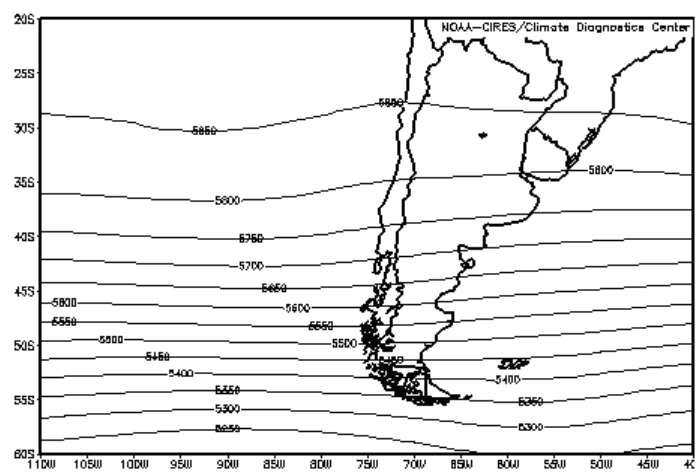

a) Campo medio de alturas geopotenciales en $500 \mathrm{hPa}$

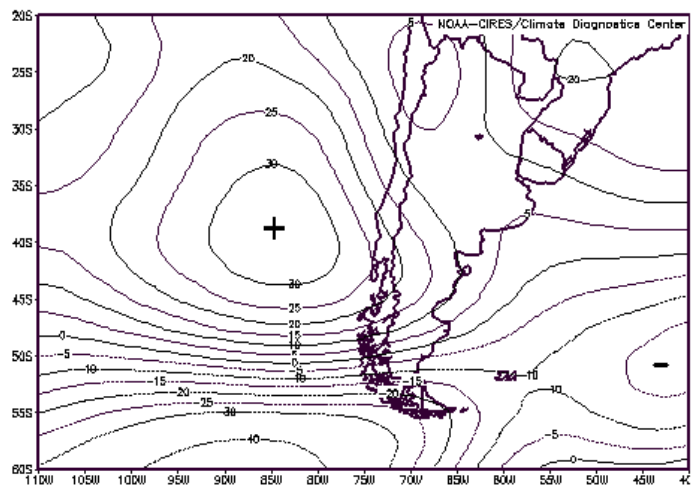

c) Anomalías de alturas geopotenciales en $500 \mathrm{hPa}$

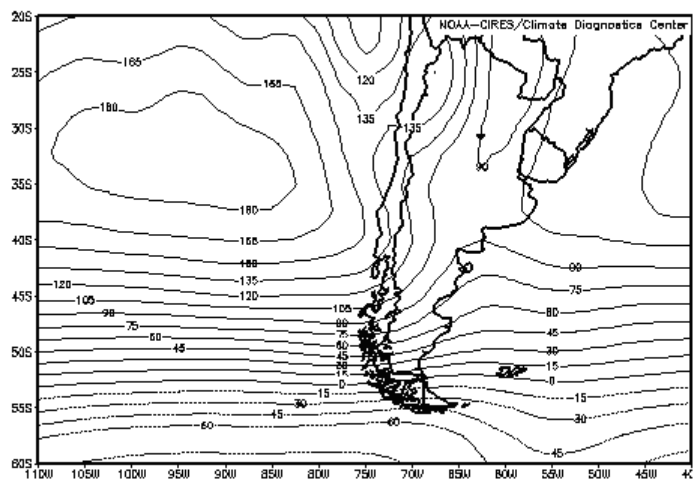

b) Idem a) pero en $1000 \mathrm{hPa}$

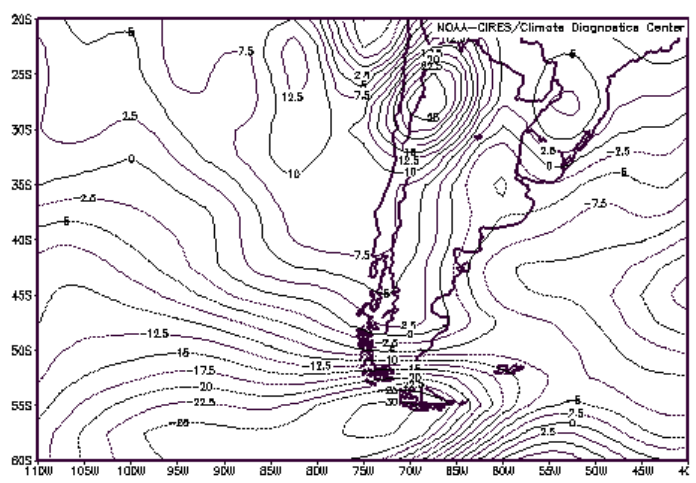

d) Idem c) pero en $1000 \mathrm{hPa}$

Figura 8 - Campos medios trimestrales de alturas geopotenciales en 500 ( a) y $1000 \mathrm{hPa}$ ( b) y sus anomalías (c y d) correspondientes al $1^{\circ}$ trim. de 2003

Para poder asociar las situaciones sinópticas de 1000 y $500 \mathrm{hPa}$ con los campos de temperatura y precipitación, se representan los campos medios de alturas geopotenciales y las anomalías de estos últimos. Los campos de cada uno de los cuatro trimestres de 2003, se muestran en las Figuras. 8, 9, 10 y 11, mientras que el correspondiente al del primer trimestre (enero, febrero y marzo) de 2004 y al anual de 2003 en la Figura 12 y 13 respectivamente.

En ambos niveles, las anomalias anuales de alturas geopotenciales en 2003, determinan valores negativos en el Pacífico y Atlántico sur (Fig. 12c y 12d), lo que indica que la circulación más frecuente en el sur de la Patagonia fue del oeste. En cambio en 2004, el Atlántico sur está afectado (Fig. 13c y d) por una anomalia positiva, que señala un debilitamiento de la circulación del oeste.

Cabe mencionar que durante la sequía producida en la Argentina en 1962 (Malaka et al, 1980), las anomalías de los campos medios de alturas geopotenciales de los dos niveles presentaron una anomalía positiva en el Atlántico sur, como la del primer trimestre de 2004. Durante la sequía de 1995(Alessandro et al, 1996) se estableció una anomalía positiva en el Pacífico, más al sur de la anomalía producida en 2003/ 2004.
En 1000 hPa (Fig. 12b), el campo anual de 2003 muestra sobre el centro y este del país y al norte de los $35^{\circ} \mathrm{S}$, un predominio de la circulación del noreste y norte, que se ve debilitado en el primer trimestre de 2004 (Fig. 13b), de acuerdo al campo de anomalias de ese nivel.

El campo medio de $500 \mathrm{hPa}$ indica en 2004 (Fig. 13a), una cuña en el centro del pais, más pronunciada al norte de los $40^{\circ} \mathrm{S}$. El correspondiente a sus anomalías se presenta totalmente diferente al anual de 2003 (Fig. 12c).

Mientras que en 2003 una anomalía positiva se halla en el Pacífico en $30^{\circ} \mathrm{S}$, en 2004 (Fig. 13c) una anomalía de igual signo se ubica en el Atlántico sur.

En consecuencia en 2003 la frecuente circulación del SO ocupa una importante región del país, mientras que en 2004 hay un fuerte debilitamiento de la circulación del oeste al sur de aproximadamente $32^{\circ} \mathrm{S}$. A pesar que los valores de las anomalías de alturas geopotenciales obtenidos en ambos niveles, son menores que las correspondientes desviaciones estándar (Alessandro, 1998; Velazco y Necco, 1980), en los próximos incisos puede verse una relación apreciable con la precipitación y en $1000 \mathrm{hPa}$ con la temperatura. 


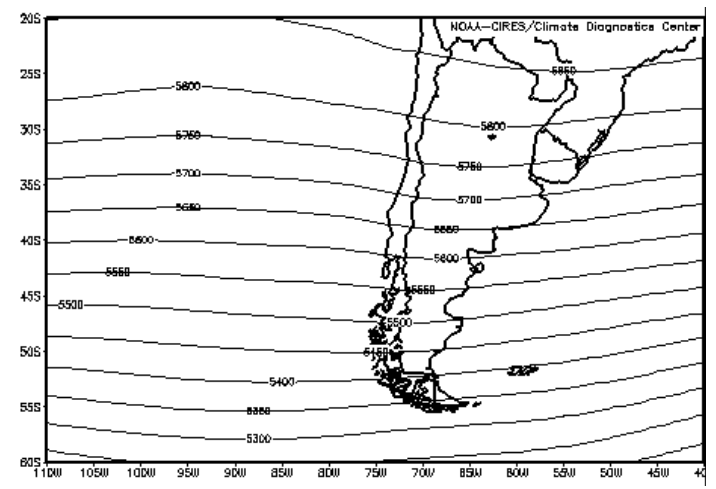

a) Campo medio de alturas geopotenciales en $500 \mathrm{hPa}$

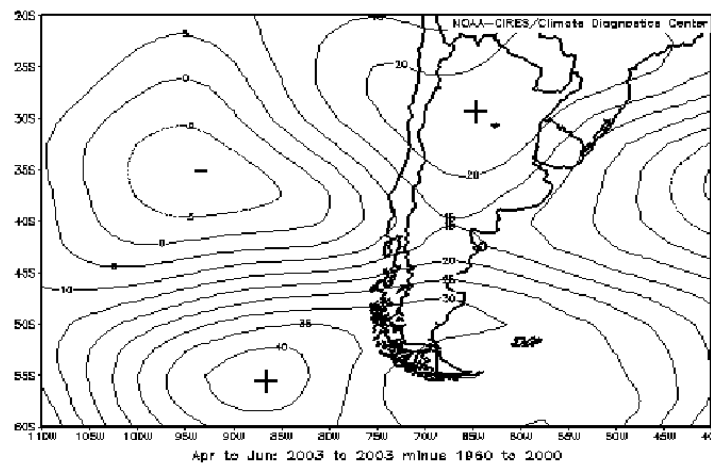

c) Anomalías de alturas geopotenciales en $500 \mathrm{hPa}$

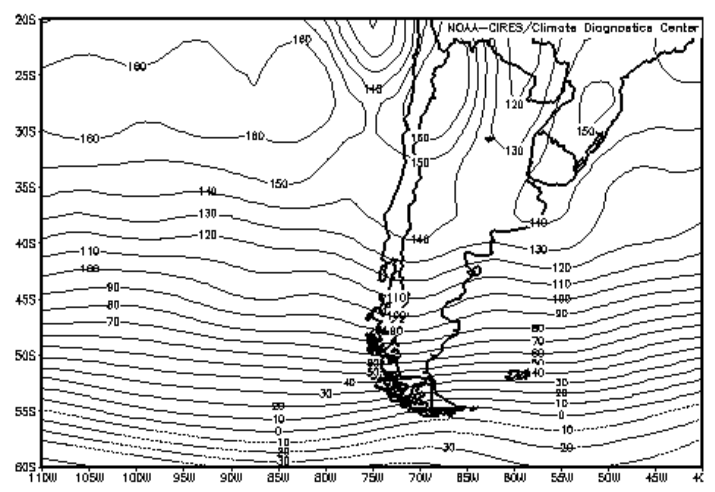

b) Idem a) pero en $1000 \mathrm{hPa}$

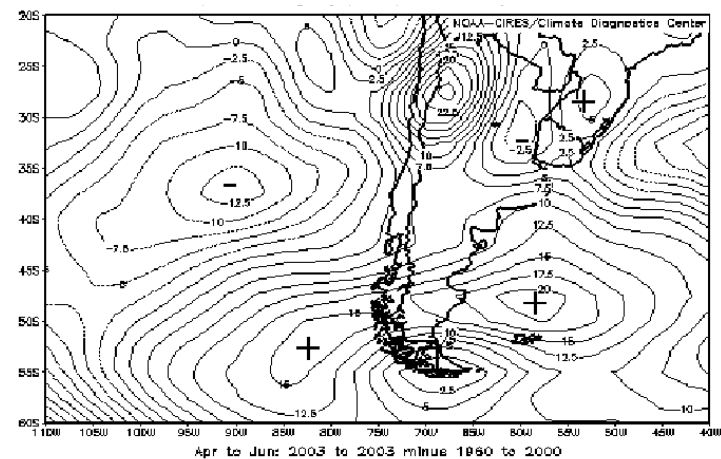

d) Idem c) pero en $1000 \mathrm{hPa}$

Figura 9 - Campos medios trimestrales de alturas geopotenciales en 500 (a) y $1000 \mathrm{hPa}$ (b) y sus anomalías (c y d) correspondientes al $2^{\circ}$ trim. de 2003.

\subsection{Asociacón entre la Precipitación y la temperatura anual de 2003 y el primer trimestre de 2004 con los campos citados en 3-1.}

Solo se hace referencia al campo anual de 2003 y al primer trimestre de 2004, pudiendo el lector con el material mostrado anteriormente, encontrar la justificación trimestral de los valores de ambas variables.

En el campo anual de 2003 (Fig. 5d) la falta de precipitación al norte de la Patagonia se debe especialmente a un flujo del $\mathrm{SO}$ en $500 \mathrm{hPa}$ (Fig. 13c), que atraviesa a laArgentina diagonalmente desde aproximadamente $45^{\circ} \mathrm{S}$ hasta los $30^{\circ} \mathrm{S}$ y a un sistema depresionario en el Océano Atlántico en $40^{\circ} \mathrm{S}$ que desplaza hacia el norte al Anticiclón del Atlántico. Esta zona se ve representada en el NO, en casi su totalidad, por las anomalías de humedad de $-3 \mathrm{~g}$ / Kg y $-4 \mathrm{~g} / \mathrm{Kg}$ en 850 y en $1000 \mathrm{hPa}$ respectivamente. Los valores de precipitación mayores al 100 $\%$ en la Patagonia, responden principalmente al pasaje de sistemas ciclónicos que se reflejan a través de las anomalías negativas en 1000 y $500 \mathrm{hPa}$ (Fig.13c y d).

La otra región con precipitaciones mayores al $100 \%$ (máximo valor de $140 \%$ ), corresponde a la formada por la Mesopotamia, centro de Santa Fe y norte y este de la provincia de Bs. As. 


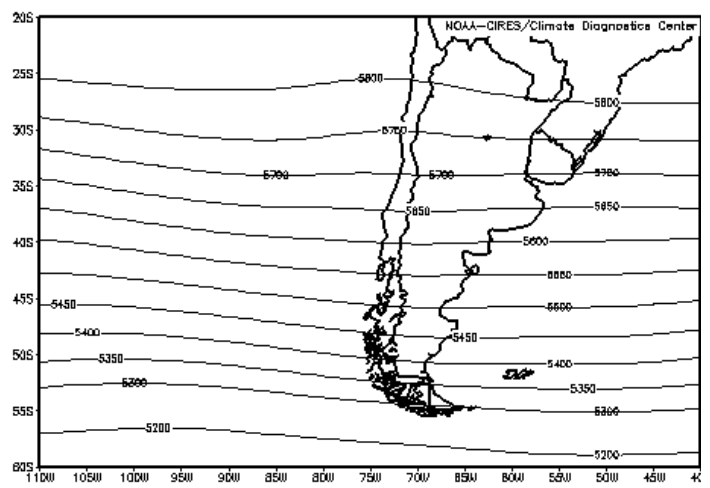

a) Campo medio de alturas geopotenciales en $500 \mathrm{hPa}$

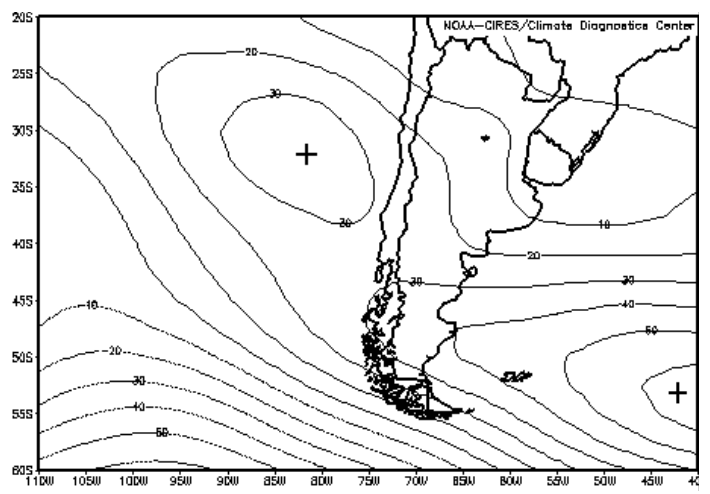

c) Anomalías de alturas geopotenciales en $500 \mathrm{hPa}$

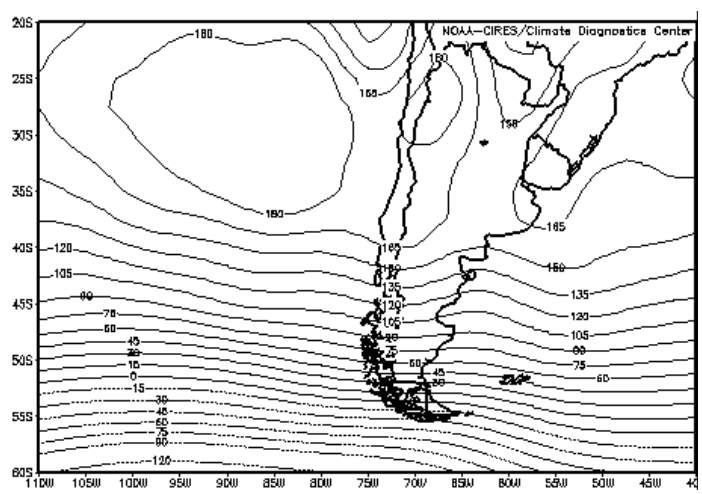

b) Idem a) pero en $1000 \mathrm{hPa}$

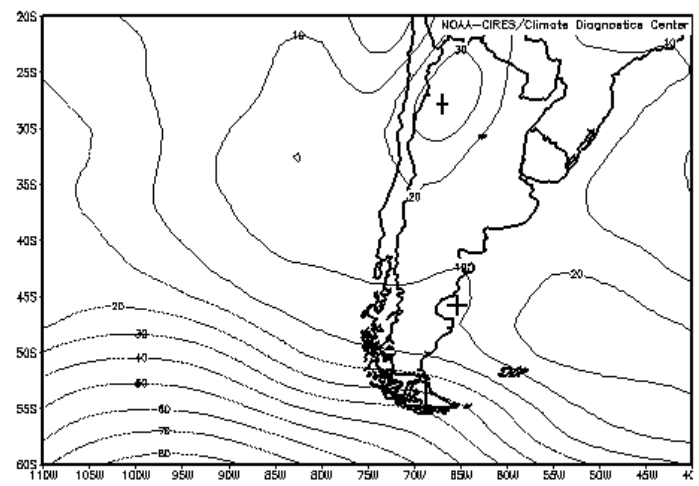

d) Idem c) pero en $1000 \mathrm{hPa}$

Figura 10 - Campos medios trimestrales de alturas geopotenciales en 500 (a) y $1000 \mathrm{hPa}$ (b) y sus anomalías (c y d)correspondientes al $3^{\circ}$ trim. de 2003.

Esta zona se halla influenciada por la parte delantera de una vaguada situada al oeste de la provincia de Bs. As. y por la circulación ciclónica del norte en 1000 hPa ( Fig. 13b) visibles también en las anomalías de este nivel (Fig. 13d). Los vientos muestran una dirección preferencial del viento $\mathrm{NE}$ al $\mathrm{NE}$ de Misiones que favorece a la humedad en la Mesopotamia.

En esta zona en general durante el año 2003, se presentaron dos ciclos lluviosos y uno deficitario. De enero a abril, las precipitaciones se mantuvieron superiores a los normales, e inferiores al norte de Misiones. De mayo a septiembre se observaron valores deficitarios (Posadas (49.4 $\mathrm{mm}$ ), Oberá (73.3 mm) y Paso de los Libres (20.6 mm), en julio) - A partir de octubre aumentaron las lluvias y en diciembre los valores volvieron a ser mayores a los normales, como por ejemplo en noviembre en Iguazú la precipitación fue de $508 \mathrm{~mm}$ frente a los $135 \mathrm{~mm}$ de su valor normal, en Corrientes fue de $351 \mathrm{~mm}$, en Posadas de $329 \mathrm{~mm}$ y en Oberá de $318 \mathrm{~mm}$.
Del análisis realizado se observa en el año 2003, que a nivel anual el ENE se vio menos afectado de la falta de precipitación que el resto del país al norte de los $40^{\circ} \mathrm{S}$, bajo la presencia de un Niño relativamente débil y una fase neutral. Bajo esta última condición, sin embargo el primer trimestre de 2004 no presentó esta característa por cuanto esta zona fue deficitaria.

El déficit producido en parte de la región húmeda y subhúmeda de la Argentina en 2003/2004 no se ha visto relacionado con el fenómeno de la Niña, que de acuerdo a Vargas y otros (1999) en esa región las sequías generalizadas están más probablemente asociadas a fases frías, si bien la ocurrencia no es biunívoca.

En el primer trimestre de 2004, lo más característico, es que a pesar de la presencia de la circulación ciclónica en el norte, con el correspondiente flujo del N y NE (Fig. 12b), en el norte predominó el déficit. 


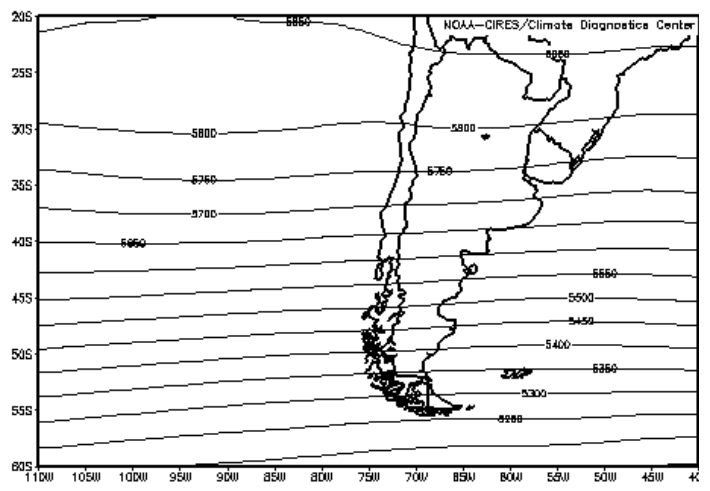

a) Campo medio de alturas geopotenciales en $500 \mathrm{hPa}$

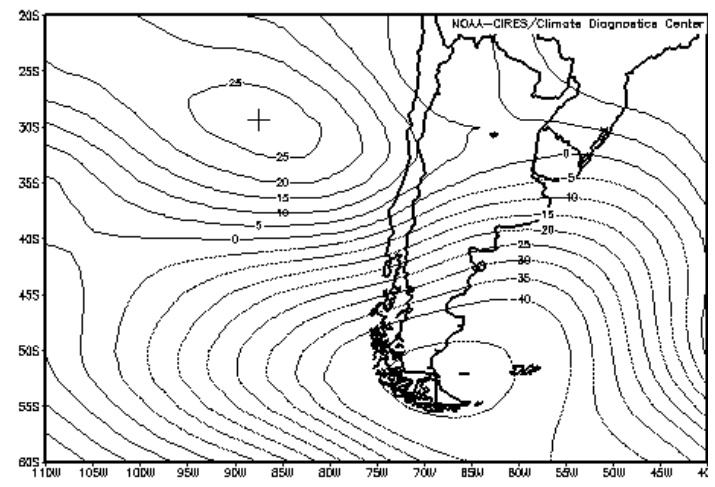

c) Anomalías de alturas geopotenciales en $500 \mathrm{hPa}$

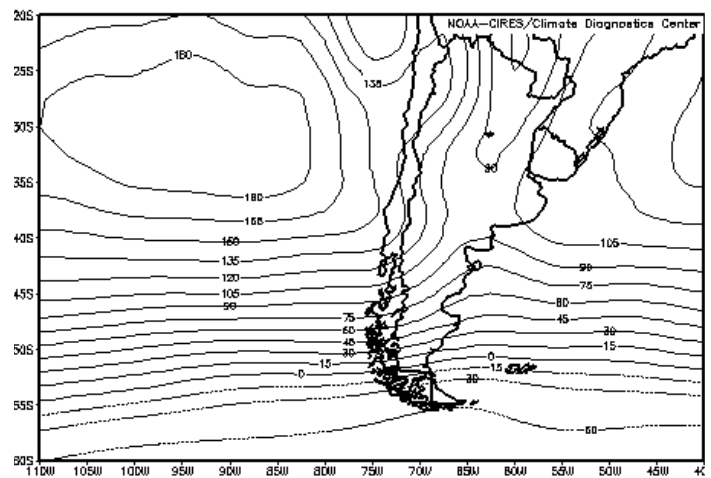

b) Idem a) pero en $1000 \mathrm{hPa}$

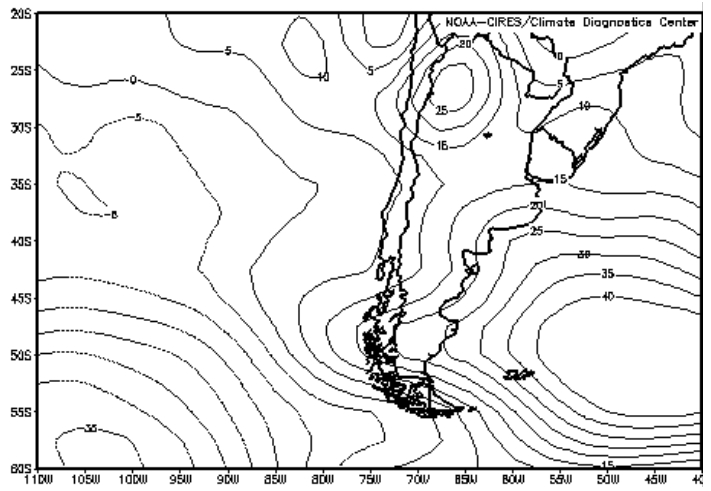

d) Idem c) pero en $1000 \mathrm{hPa}$

Figura 11 - Campos medios trimestrales de alturas geopotenciales en 500 (a) y $1000 \mathrm{hPa}$ (b) y sus anomalías (c y d) correspondientes al $4^{\mathrm{o}}$ trim. de 2003.

Esta característica fue producida por la cuña en $500 \mathrm{hPa}$, que se ubica en aproximadamente $\operatorname{los} 67^{\circ} \mathrm{O}$, desde los $25^{\circ} \mathrm{S}$ hacia el sur, que se pone también de manifiesto a través de una gran anomalía positiva con centro en el Atlántico sur (Fig. 12c) y que se detecta también en $1000 \mathrm{hPa}$. La gran extensión que ocupa dicha anomalía indica una disminución de las perturbaciones provenientes del oeste en estas latitudes que favorecen también al déficit en gran parte de la Patagonia. La costa de ésta última no se presenta deficitaria debido a la circulación prevaleciente desde el Atlántico al sur de los $30^{\circ} \mathrm{S}$ (Fig. $12 \mathrm{~d}$ ).

Las variaciones de la precipitación del primer trimestre de 2004 se ven muy reflejadas en el campo de humedad específica de 850 y $1000 \mathrm{hPa}$ (Fig. 4f), donde los valores positivos de $\Delta \mathrm{q}$ coinciden con la diagonal donde se produjeron las mayores precipitaciones. El campo anual de temperatura de
2003 (Fig. 5f) muestra valores positivos en toda la Argentina y con máximos valores en la zona próxima a la diagonal que se extiende desde los $25^{\circ} \mathrm{S}$ y $70^{\circ} \mathrm{O}$ hasta $\operatorname{los} 40^{\circ} \mathrm{S}$ y $65^{\circ} \mathrm{O}$. Al sur de los $40^{\circ} \mathrm{S}$ se observa a partir de las anomalías de $1000 \mathrm{hPa}$ (Fig. 13d) el flujo predominante del NO que aporta valores positivos a esta zona.En el primer trimestre de 2004, los desvíos de la temperatura media fueron positivos en todo el país; mayores a $1^{\circ} \mathrm{C}$ en su mayor parte, a excepción del este y centro del mismo, al norte de los $34^{\circ} \mathrm{S}$. Se puede observar el flujo prevaleciente desde el noreste del país y desde el Atlántico al sur de los $35^{\circ} \mathrm{S}$ (Fig. 12d y Fig. 3f), ambos causantes del mayor calentamiento del centro oeste y al sur de los $35^{\circ} \mathrm{S}$.

Las anomalías mayores a $1^{\circ} \mathrm{C}$ coinciden en ambos años (Fig. 5e y f), en una diagonal que se extiende entre los $42^{\circ}$ y $30^{\circ} \mathrm{S}$. 


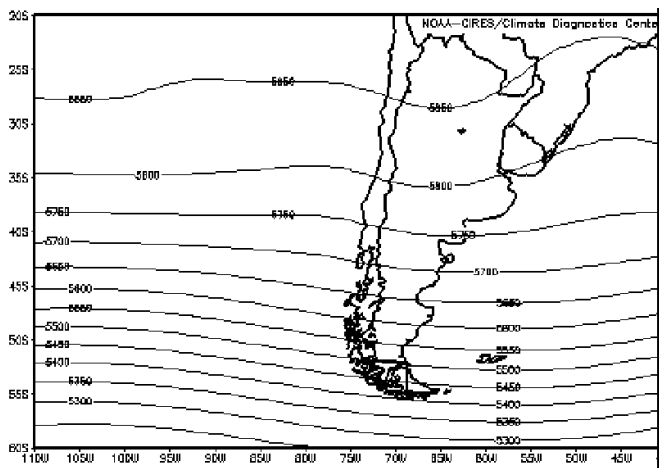

a) Campo medio de alturas geopotenciales en $500 \mathrm{hPa}$

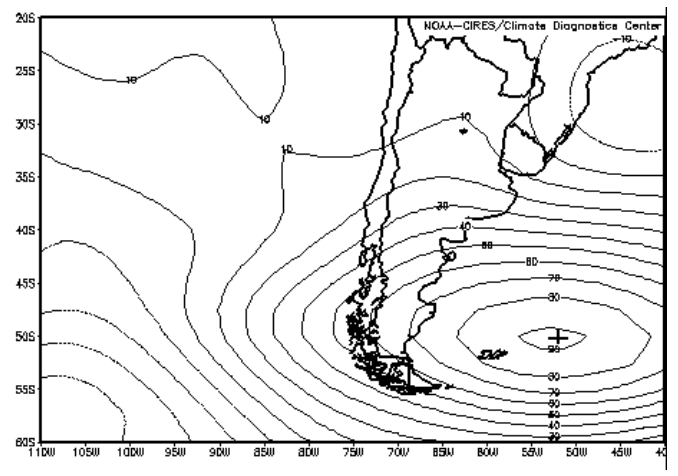

c) Anomalías de alturas geopotenciales en $500 \mathrm{hPa}$

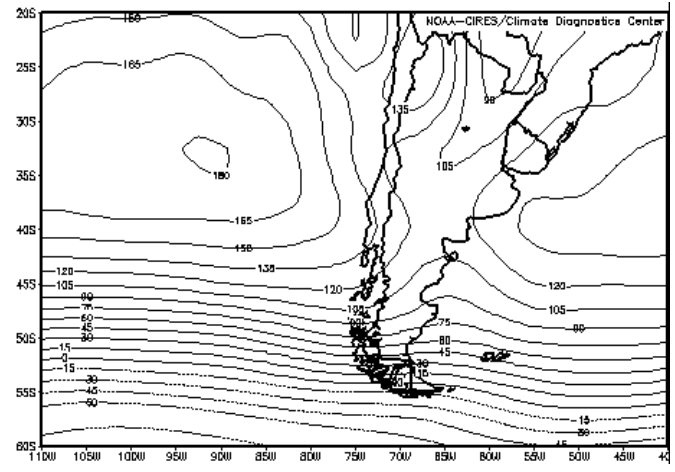

b) Idem a) pero en $1000 \mathrm{hPa}$

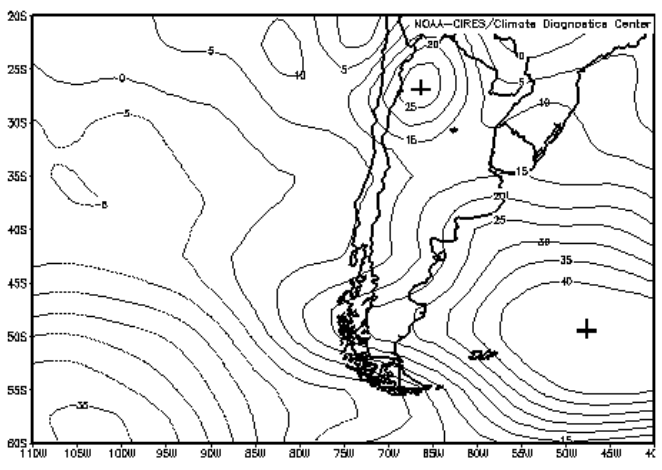

d) Idem c) pero en $1000 \mathrm{hPa}$

Figura 12 - Campos medios trimestrales de alturas geopotenciales en 500 (a) y $1000 \mathrm{hPa}$ (b) y sus anomalías (c y d) correspondientes al $1^{\circ}$ trim. de 2004.

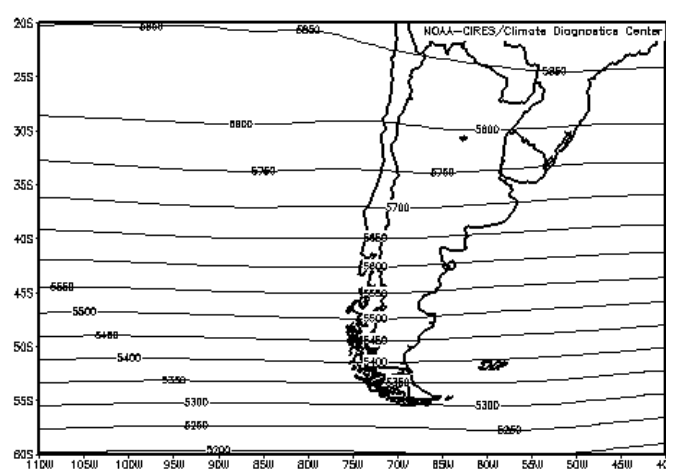

a) Campo medio de alturas geopotenciales en $500 \mathrm{hPa}$

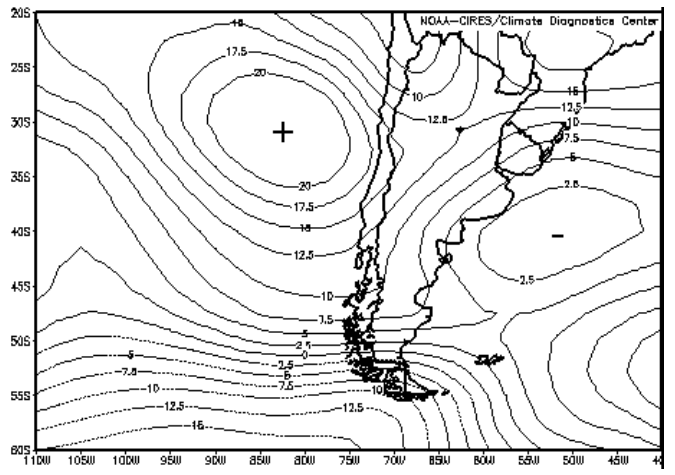

c) Anomalías de alturas geopotenciales en $500 \mathrm{hPa}$

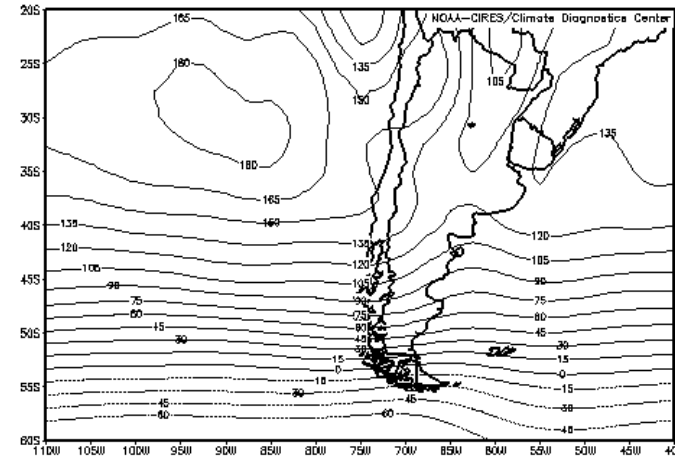

b) Idem a) pero en $1000 \mathrm{hPa}$

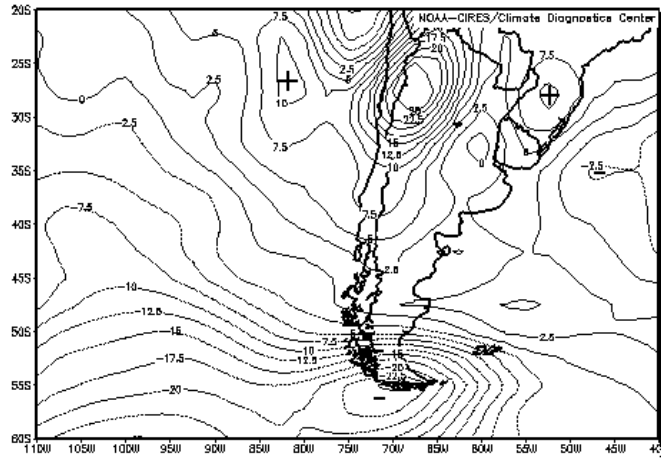

d) idem c) pero en $1000 \mathrm{hPa}$

Figura 13 - Campos medios anuales de alturas geopotenciales en 500 (a)y $1000 \mathrm{hPa}$ (b) y sus anomalías (c y d) correspondientes a 2003. 


\section{CONCLUSIONES}

En este trabajo se estudio la circulación atmosférica durante la intensa sequía que afecto gran parte de Argentina el año 2003 y parte del 2004. Las principales conclusiones son:

I. Al norte de los $40^{\circ} \mathrm{S}$, el campo porcentual medio de precipitación de 2003, muestra al este argentino menos afectado de la falta de precipitación que el resto del país, bajo la presencia de un Niño relativamente débil y una fase neutral. El correspondiente al primer trimestre de 2004 bajo esta última fase, presenta en la misma zona condiciones inversas de precipitación. En todo el período considerado, las principales precipitaciones en el país al norte de los $40^{\circ} \mathrm{S}$ son mayormente consecuencia de la dirección predominante del viento del sector este. En la Patagonia se hallan asociadas a un número mayor de casos de advección de aire húmedo desde el Atlántico y con menor frecuencia al flujo del NO desde el Pacífico.

II. Las causas principales de la falta de precipitación del primer trimestre de 2004 se debe a la presencia de una cuña en $500 \mathrm{hPa}$ ubicada en el centro norte del país y a la frecuente circulación anticiclónica en el Atlántico Sur al este de las Islas Malvinas que impide el normal desplazamiento hacia el este, de las depresiones en esas latitudes. El campo medio de humedad específica asociado, presenta valores negativos al norte de los $35^{\circ} \mathrm{S}$.

III. Regionalmente las anomalías de alturas geopotenciales en $500 \mathrm{hPa}$, muestran un flujo del SO que atraviesa al país diagonalmente desde aproximadamente $45^{\circ} \mathrm{S}$ hasta los $30^{\circ} \mathrm{S}$ y un sistema depresionario en el Océano Atlántico en $40^{\circ} \mathrm{S}$ que desplaza hacia el norte al Anticiclón del Atlántico, lo aleja del continente y lo debilita. Asociada a esta situación y por ende a la falta de precipitación al norte del Río Colorado en 2003, se presentan en el nivel de $850 \mathrm{hPa}$, valores de anomalías negativas de humedad $(\Delta \mathrm{q})$ al norte de los $40^{\circ} \mathrm{S}$, más intensas durante el primer y cuarto trimestre.

IV. En la escala hemisférica, se observan en $500 \mathrm{hPa}$, dos centros de anomalías positivas de altura geopotencial. El de menor intensidad y de posición más variable sobre las costas chilenas se asocia a la prolongada sequía de al menos 15 meses.

V. Las anomalías de temperatura del período 2003-2004 fueron positivas en todo el territorio argentino. En 2003 al norte de los $48^{\circ} \mathrm{S}$, de acuerdo a la situación sinóptica de $1000 \mathrm{hPa}$, se debe en primer lugar a la subsidencia ocasionada por el Anticiclón del Pacífico desplazado hacia el este de su posición normal y a una mayor subsidencia al este de la cordillera de los Andes; y en segundo lugar al aporte del flujo de dirección $\mathrm{N}$ y NE. Al sur de esta última latitud como consecuencia de la subsidencia orográfica producida por la intensa circulación del oeste. En el primer trimestre de 2004 se debe al flujo N desde el norte del país y a la frecuente circulación anticiclónica en el Atlántico al sur de los $35^{\circ} \mathrm{S}$.

\section{AGRADECIMIENTOS}

La autora agradece las invalorables sugerencias del Dr. Erich R. Lichtenstein, profesor consulto del Dto. de Cs. de la Atmósfera de la Universidad de Buenos Aires y a ésta última que a través del subsidio X234 ha financiado este trabajo.

\section{REFERENCIAS BIBLIOGRÁFICAS}

Aceituno, P.: On the functioning of the Southern Oscillation in the South American sector, 1, Surface climate, Mon. Weather Rev., 116, p 505-424, 1988.

Alessandro A. P.: Contribuciones al estudio de la Climatología sinóptica en la Argentina. Tésis Doctoral. UBA, p 8-12, 1998.

Alessandro A. P., Lichtenstein R. E.: Situación sinóptica asociada a la sequía del invierno de 1995. Revista Geofísica, IPGH. 45, p 125-1443, 1996.

Alessandro A. P.: Aspectos sinópticos de las intensas lluvias del verano 82-83. Meteorologica 23 (1 y 2), p 1-14, 1999.

Alessandro A. P.: Análisis of the heavy precipitations during the summer 1997-1998 in southeastern South America and their relations with the summer 1982-1983. Atmósfera, 14, p 189-202, 2001.

Antico P. L.: Búsqueda de patrones entre las anomalías mensuales de geopotencial y precipitación en la región pampeana. Tesis de Licenciatura, Departamento de Ciencias de la Atmósfera. FCEyN. Universidad de Buenos Aires, $\mathrm{p}$ 8-60, 1998

Diaz A. F., Studzinski C. D., Mechoso C. R.: Relationships between precipitation anomalies in Uruguay and Southern Brasil and sea surface temperature in the Pacific and Atlantic Ocean. Journal of Climate, 11, p 251-271, 1998.

Fernandez A., Necco G.: Características del campo del viento en la atmósfera libre en estaciones argentinas. Meteorologica XIII, 2 , p 7-21, 1982.

Fernández A.: Estudio de la circulación y humedad troposférica en áreas argentinas. Tesis Doctoral. UBA. Parte 1: Circulación troposférica regional. Pags. 33-34. Parte II: Humedad troposférica regional, p 3-14, 2004.

Fontana D. C., Grimm M. A.: Influencia do El Niño OscilaVao Sul sobre a precipitaVao Pluvial no Estado do Río Grande do Sul. Revista Brasileira de Agrometeorologia, Santa María, 5, 1, p 127-132, 1997.

Grimm A. M., Silva Dias P. L.: Sea surface temperatures in the Pacific and rainfall over part of Southern Brasil. Part 1: Correlations. Ann. Acad. Bras. Cienc, 68, p 3-9, 1996. 
Grimm A. M., Silva Dias P. L.: Sea surface temperatures in the Pacific and rainfall over part of Southern Brasil. Part 2: Dynamical mechanisms. Ann Acad. Bras. Cienc., 68, p 11-16, 1996.

Grimm, A. M., Barros V. R., Doyle M. E.: Climate variability in southern South America associated with El Niño and La Niña event, J. Clim, 13(1), p 35-58, 2000.

Inzunza J. C. : Estudio de la Corriente en Chorro en capas bajas en el Norte de Argentina. Tesis Doctoral. UBA, p 39-42, 1992.

Gordillo S. B.: Estudio de las líneas de inestabilidad en la República Argentina. Tesis Doctoral UBA, p 8-84, 1986.

Lichtenstein R. E.: La Depresión del Noroeste argentino. Tesis doctoral UBA, p 10-50, 1980.

Malaka I., Nuñez S.: Aspectos sinópticos de la sequía que afectó a la República Argentina en 1962. Geoacta 10, p 1-22, 1980.

Minetti J. L., Vargas W. M., Poblete A.: Comportamiento del borde oriental del Anticiclón del Pacífico. Geofísica, 38, p 79-89, 1993.

Nogues-Paegle, J., Mo K. C.: Alternating wet and dry conditions over South America during summer. Mon. Weather. Rev., 125, 2, p 79-291, 1997.

Pisciottano G., Díaz A. F., Cazes G., Mechoso R. C.: El NiñoSouththern Oscilation impact on rainfall in Uruguay. J. Clim. 7, p 1286-1302, 1994.

Ropelewski C. F., Halpert M. S.: Global and regional scale precipitation patterns associated with the El Niño-Southern Oscillation, Mon. Weather Rev, 115, p 1606-1626, 1987.
Ruiz, N. E., Vargas W. M.: 500 hPa Vorticity Analyses over Argentina: Their Climatology and Capacity to distinguish Synoptic-Scale Precipitation, Springer Wien New York, Austria. 2, Theoretical and Applied Climatology, 60, p 77-92, 1998.

Ruiz, N. E.: Vorticity patterns for rainy and dry episodes in Argentina, , Springer Wien New York, Austria, . Meteorology and Atmospheric Physics, 86, p 45-62, 2004.

Rutllant, J., Fuenzalida H.: Synoptic aspects of the central Chile rainfall variability associated with the Southern Oscillation, Int. J. of Climatology, 11, p 63-76, 1991.

Salio P.: Caracterización de Eventos de Corriente en Chorro en Capas Bajas de la Atmósfera en base a Reanálisis y la precipitación asociada en el Sudeste de Sudamérica. Tesis Doctoral. UBA. P 3-70, 2002.

Showalter A.: A stability index for thndetstorm forecasty. Amer. Met. Sc. 6, p 240-252, 1953.

Trenberth, K. E.: The definition of El Niño, Bull. Amer. Meteor. Soc., 78, p 2771-2777, 1997.

Vargas W. M., Penalba O., Minetti J.: Las precipitaciones mensuales de la Argentina y el ENOS. Un enfoque hacia el problema de la decisión. Meteorológica 24, p 3-18, 1999.

Velasco I., Necco G. V.: Valores medios, extremos y desviaciones Estandar de datos aerológicos de la República Argentina. Universidad de Buenos Aires.FCEN. Dto. de Meteorología, 1980 . 